SCIREA Journal of Economics

http://www.scirea.org/journal/Economics

September 28, 2021

SCIREA

Volume 6, Issue 4, August 2021

\title{
Analysis of Market Equilibrium based on the New Theory of Value
}

\author{
Jie $\mathbf{W u}^{1}$, Zili $\mathbf{W u}^{2, *}$ \\ ${ }^{1}$ Development Research Institute, Shandong University, China \\ ${ }^{2}$ Guangzhou Milestone Software Co., Ltd., China \\ *Corresponding author
}

\begin{abstract}
Based on the axiomatic assumptions that the law of diminishing marginal utility, non-satiation assumption and social choice assumption based on personal preference, Arrow and Debreu study general equilibrium problems whose scope of research only involves the quantity, utility and price of commodities. Therefore, the conclusion can only explain the phenomenon of general equilibrium in the process of quantitative change, but not the non-equilibrium phenomenon in the qualitative change process. In view of the above problems, this paper will expand the research content to the range of quantity, quality, time, labor value, use value, value, exchange value and price based on the new theory of value, to comprehensively analyze the phenomenon of quantitative change and qualitative change in the process of commodity exchange, give the mathematical explicit and general solution of the unified price function of equilibrium and non-equilibrium, and prove the existence, uniqueness and stability of extremum solutions of various price functions. The purpose of this paper is to reveal the general economic law in the process of commodity exchange. At the same time, it will also briefly analyze why the general equilibrium theory can cause the errors of incompleteness, incompatibility and cyclic argumentation.
\end{abstract}


Keywords: new theory of value, value, price, metric, fixed point, market equilibrium

\section{Introduction}

The general equilibrium theory was founded by Walras. According to the law of diminishing marginal utility, Walras chooses one of $\mathrm{n}$ commodities to act as "the universal equivalent" to measure the value of other commodities, reduces the price of a commodity to the proportional relationship between the quantity of one currency and the quantity of other n-1 commodities, and establishes a general equilibrium price simultaneous equation of both supply and demand sides formed by $2 \mathrm{n}-1$ equations to solve the market equilibrium price. (Walras, 1874, p.365-367) Further, Walras believes that, since the aggregate amount of prices of commodities sold in the market is constantly equal to the aggregate amount of prices of commodities purchased that is Walras law. Therefore, the simultaneous equations of general equilibrium prices which satisfy the constraint conditions of Walras law usually have solutions. Meanwhile when there is an equilibrium price, there must be the balanced relation between supply and demand of commodities. (Walras, 1874, p.40-41)

However, Walras's general equilibrium theory has defects, including failing to strictly prove that the utility of commodities is measurable on the basis of cardinal utility theory (Hicks \& Allen, 1934), and not proving the existence and uniqueness of the solution of the general equilibrium price equation (Arrow, Debreu, 1954). In order to solve these problems, many economists have made efforts. The most influential Neoclassical solutions were given by Pareto, Hicks, Nash, Arrow and Debreu. Pareto (1906) first used indifference curve to analyze general equilibrium theory and constructed Pareto optimal utility function based on partial order relation. Hicks and Allen (1934) further proposed to adopt ordinal utility theory instead of Walras and Jevons' cardinal utility theory. Nash (1951) believed that the economic relationship in the process of Commodity Exchange was essentially a game relationship, and there must be a Nash equilibrium under the dominant strategy.

According to the logic of the ordinal utility theory, Arrow (1951) inferred the impossibility theorem, which denied the conclusion that utility could be summed over and the utility function could have a global optimal solution in cardinal utility theory. Further, on the basis of the ordinal utility theory and the impossibility theorem, Arrow and Debreu (1954) founded an axiomatic assumption system of social contract based on democratic system, and accordingly gave the continuity theorem of utility function which satisfies the order of 
consumers' personal preference. Especially, all the general equilibrium theory based on ordinal utility theory only has the extremum solutions of local optimization, instead of the global maximum solution. Also, according to Nash equilibrium theory, Arrow and Debreu (1954) inferred that under the condition of sufficient market competition, spontaneous market game behavior must lead to a general equilibrium satisfying Pareto optimality.

In conclusion, there are various internal logical contradictions in general equilibrium theory:

(1) From the perspective of pure mathematical logic, it is needed to define the utility function based on Euclidean metric space to prove the existence of equilibrium price fixed point strictly. (Debreu, 1959, p.12, p.36) If the commodity space is not Euclidean metric space, then it is unable to established for all kinds of conclusions drew from general equilibrium theory analyzed with point set topology and convex analysis theory by Debreu. However, if the given Euclidean metric is the norm of commodity space, then the commodity space has the property of total order. This conclusion is obviously contradictory to Hicks' ordinal utility theory and Arrow's impossibility theorem.

(2) In order to prove the continuity of utility function, the general equilibrium theory must ensure that there can be no overcapacity. Therefore, Arrow and Debreu (1954) regard "insatiable demand" as a basic axiomatic assumption. However, this is obviously inconsistent with the facts. Under the condition that the quality of the product remains unchanged, any individual's demand for the quantity of any product has an upper bound point. Beyond the upper bound point, the increased quantity of commodities has no value. (Wu, et al., 2020) Therefore, under the acting force of "learning by doing" (Yang \& Borland, 1991), the production of the same product with constant quality for a long time, will inevitably lead to the phenomenon of overcapacity and corresponding diminishing marginal value. The general equilibrium theory assumes that diminishing marginal utility and insatiable demand are basic axioms, which inevitably leads to the contradiction of imperfection, that is, it can only explain the special economic law of commodity price in the process of quantitative change, but cannot explain the law of price deviating from value in the non-equilibrium state in the process of qualitative change.(Arrow \& Debreu, 1954) Theorem 5.4 in this paper will prove the above conclusion.

(3) The first theorem and the second theorem of welfare economics are both locally valid, that is, they can only be valid in a slight unbalanced state of supply and demand. In the period of economic crisis, the phenomenon of structural overcapacity appears. The partial order relationship based on individual preference and Nash equilibrium can only lead to vicious 
competition, so that most people to make correct decisions for global optimal under the guidance of scientific economic theory. In this paper, Theorem 5.2 and 5.3 will prove that the global equilibrium is different from the local equilibrium. Therefore, in the stages with different levels of overcapacity, although the full competitive market can make the trade benefits reach the maximum at this stage, that is, the Nash equilibrium distribution of any group of competitive markets must be Pareto efficient allocation. However, under the Arrow-Debreu definition, Pareto collocation is a kind of local optimal extremum solutions based on partial order relation, not necessarily a global maximum solution. Therefore, neither the first theorem nor the second theorem of welfare economics can guarantee the economic system to get out of the economic crisis caused by overcapacity. In fact, there is an inverse relationship between the quantity, quality and value of commodities ( $\mathrm{Wu}$, et al., 2020). If we understand the theorem that commodity quality is inversely proportional to labor productivity, we can eliminate excess capacity by expanding the production scale of high-quality products in case of overcapacity, and restore the balance between market supply and demand of commodities at a high quality level. This conclusion will be proved by theorem 2.1 in this paper.

In view of the above problems, this paper studies the general equilibrium from the cardinal utility theory. We accept the following basic axiomatic assumptions: (1) labor determines value (Smith, 1776, p.15); (2) there is abstract and general measure of value; (Marx, 1867, ch.1) (3) labor value and use value can be converted to each other (Jevons, 1875, p.170-209); (4) labor creates surplus value (Marx, 1867, Pt.III); (5) the equilibrium price of every commodity exists objectively, price is equal to everyone. Although everyone can have different evaluation standards for the same commodity, the probability distribution of personal preference has unique utility value under linear transformation, and makes the market price of commodities tend to the equilibrium price (Neumann, Morgenstern, 1953, ch.3-4). At the same time, personal preferences will change at different stages of the economic cycle. Thus only the analysis of personal preferences must be combined with the overall value judgment is comprehensive and objective; (6) there is an upper bound point for human natural demand (Aristotle, p13-14). Obviously, the above axiomatic hypothesis has a strict definition supplemented and improved in the basic axiomatic hypothesis system of the new theory of value. Therefore, we can start from the cardinal utility theory accordingly, construct the Euclidean value metric space of commodity on the new theory of value, and thoroughly investigate the market equilibrium problem of commodity price under the condition of 
quantitative change and qualitative change by using complex variable function theory, linear normed space theory and convex analysis theory.

To sum up, this paper will comprehensively analyze the problem of market equilibrium based on the new theory of value. Here, theoretical research involves many problems, including value concept, measure of value, value calculation, exchange value, equilibrium price, market price, hyperlane, convex function, convex set, convex hull, fixed point and so on. At the same time, in order to make it easier to understand, we will try to make a comparative analysis with the mainstream general equilibrium theory in this paper.

\section{Theory of Commodity Value in Complex Number Field}

According to the new theory of value, generally the value function of a commodity will be expressed as two dual ternary quadratic compound functions. Furthermore, if we regard the labor value function and use value function of commodities as continuous differentiable functions in the cyclic process of repetitive production and consumption, then we can express the value function of commodities in the form of mathematical theory of complex variable function. In this case, we will be able to comprehensively examine the relationship between the value and the quantity, quality, labor value and use value of commodities at the same time.

\subsection{Compound Function of the Value of a Commodity}

In general, the value function of a commodity is as follows

$$
w=\omega\left(\hat{w}(t), \hat{w}^{*}(t)\right)
$$

where $w \in W \cong R$ is the value of commodities, $\omega$ is the value function of commodities, $t$ is the time consumed in the commodity production process, $\hat{w}$ is the labor value of commodities, $\hat{w}^{*}$ is the use value of commodities, among which the labor value is

$$
\hat{w}=\hat{w}_{1}+\hat{w}_{2}=\phi\left(f_{1}\right)+\varphi\left(f_{2}\right)=\phi(\hat{m} a)+\varphi(\widehat{b u})=\phi\left(\hat{m} \frac{d^{2} b}{d t^{2}}\right)+\varphi\left(\widehat{b} \frac{d^{2} m}{d t^{2}}\right)
$$

where $\hat{w}_{1}$ is the labor value related to quantity, $\hat{w}_{2}$ is the labor value related to quality, $b$ is the quantity in the commodity production process when quality is constant, $m$ is the quality in the commodity production process when quantity is constant, $\hat{b}$ is the quantity coefficient in the process of the labor-power doing work, $\widehat{m}$ is the quality coefficient in the process of the labor-power doing work; and the use value is 


$$
\hat{w}^{*}=\hat{w}_{1}^{*}+\hat{w}_{2}^{*}=\phi^{*}\left(f_{1}^{*}\right)+\varphi^{*}\left(f_{2}^{*}\right)=\phi^{*}\left(\widehat{m}^{*} a^{*}\right)+\varphi^{*}\left(\widehat{b}^{*} u^{*}\right)=\phi^{*}\left(\widehat{m}^{*} \frac{d^{2} b^{*}}{d t^{2}}\right)+\varphi^{*}\left(\widehat{b}^{*} \frac{d^{2} m^{*}}{d t^{2}}\right)(2-1-3)
$$

where $\hat{w}_{1}^{*}$ is the use value related to quantity, $\hat{w}_{2}^{*}$ is the use value related to quality, $b^{*}$ is the quantity in the commodity consumption process when quality is constant, $m^{*}$ is the quality in the commodity consumption process when quantity is constant, $t^{*}$ is the time parameter in the commodity consumption process, $\widehat{b}^{*}$ is the quantity coefficient in the process of the consumption-power doing work, $\widehat{m}^{*}$ is the quality coefficient in the process of the consumption-power doing work.

By analyzing the above equations (2-1-1), (2-1-2) and (2-1-3), we can see that the compound function of commodity value has three characteristics:

First, the definition domain of commodity value function consists of three basic quantities: quantity, quality and time;

Second, the value of commodities is determined by displacement acceleration of commodity combination points in commodity space in the process of production and consumption, that is, labor value function and use value function are the result of the calculation of time derivation by the quantity and quality of commodities;

Third, the labor value and the use value of commodities are a dual relationship.

Therefore, the commodity value function is expressed by two binary double ordinary differential equations with one positive and one negative.

\subsection{Complex Variable Function of the Value of $\mathbf{n}$ Commodities}

Suppose that in a closed market economy system, on the one hand, people create the labor value of commodities in the process of production; on the other hand, it also realizes the use value of commodities in the process of consumption. Therefore, under the premise that the value of commodities is composed of labor value and use value, we can regard the above economic relations as a process of repetitive value circulation, and express the value relations of commodities through complex variable function. In particular, if the definition domain of complex variable function of value is a simply connected region, it means that human beings can consume all subsistence products and realize all their use value in this region without any external conditions, as long as they use their own instincts. If it is not a simply connected domain, the situation will be more complicated. In order to simplify the discussion, in this 
paper, we only study the complex variable function of value in simply connected domains. In addition, we will take time which is one of the three factors that determine value - quantity, quality, and time - as constant, ${ }^{[1]}$ that is, value is seen as a quantity determined by uniform acceleration. ${ }^{[2]}$

Definition 2.1 It is assumed that the value of a commodity is determined by uniform acceleration. Therefore, according to (2-1-1), (2-1-2) and (2-1-3), the value function of any $\alpha$ th commodity among $\mathrm{n}$ commodities can be expressed as

$$
\begin{aligned}
f_{\alpha}=m_{\alpha} a_{\alpha}+b_{\alpha} u_{\alpha} \rightarrow f_{\alpha} & =m_{\alpha} \bar{a}_{\alpha}+b_{\alpha} \bar{u}_{\alpha} \\
& =m_{\alpha} \frac{v_{\alpha t_{1}}-v_{\alpha t_{0}}}{t_{1 \alpha}-t_{0 \alpha}}+b_{\alpha} \frac{r_{\alpha t_{1}}-r_{\alpha t_{0}}}{t_{1 \alpha}-t_{0 \alpha}} \\
& =m_{\alpha} \frac{\frac{d b_{\alpha}}{d t_{1 \alpha}}-\frac{d b_{\alpha}}{d t_{0 \alpha}}}{t_{\alpha}}+b_{\alpha} \frac{\frac{d m_{\alpha}}{d t_{1 \alpha}}-\frac{d m_{\alpha}}{d t_{0 \alpha}}}{t_{\alpha}}
\end{aligned}
$$

where $\alpha=1,2, \cdots, n, t_{0}$ and $t_{1}$ represent the initial time and the final time respectively, $\bar{a}_{\alpha}$ and $\bar{u}_{\alpha}$ represent the uniform acceleration related to quantity and the uniform acceleration related to quality respectively. Obviously, (2-2-1) satisfies the theoretical proposition that the labor-power determines the value, and simplifies the discussion.

Definition 2.2 Let the quantity and quality space of any $\alpha$ th commodity be $G_{\alpha}^{2}=B_{\alpha} \times M_{\alpha}, B_{\alpha}, M_{\alpha} \cong R$, and $R$ is the set of one-dimensional real numbers. Given the point set $C_{\alpha} \subset G_{\alpha}^{2}$, the distance of the displacement on $\forall z_{\alpha}=\left(b_{\alpha}, m_{\alpha}\right) \in C$ represents the value generated under the action of the labor-power and consumption-power, then the value complex can be expressed as

$$
w_{\alpha}=F\left(z_{\alpha}\right)=\hat{w}\left(b_{\alpha}+m_{\alpha}\right)+i \hat{w}^{*}\left(b_{\alpha}+m_{\alpha}\right)
$$

where $\left|w_{\alpha}\right|=f\left(z_{\alpha}\right)=\sqrt{b_{\alpha}^{2}+m_{\alpha}^{2}}$, that is value $w_{\alpha}$ is the mode of vector $z_{\alpha}$.

Then, according to the (2-1-1), (2-1-2), (2-1-3), the complex number of labor value of any $\alpha$ th commodity is denoted as $\hat{w}_{\alpha}=b_{\alpha}+i m_{\alpha}$, all of which expressed by physical form - the

[1] If we take time as a variable, the scope of our research will be extended to non Euclidean metric spaces, such as Riemannian metric spaces. Please refer to the discussion on the differential fiber bundle of wealth value in On Wealth $(\mathrm{Wu}, 2006,2012)$ for your interest.

[2] Marx (1867, ch.1, sec.1) regarded value as the amount determined by the average labor-time socially necessary. 
quantity $b_{\alpha}$ and quality $m_{\alpha}$ of the product - has corresponding complex number related to the use value $\hat{w}_{\alpha}^{*}=b_{\alpha}+i m_{\alpha}$. The use value $\hat{w}_{\alpha}^{*}$ is called the function of the corresponding point $\hat{w}_{\alpha}$ of labor value, and the corresponding point $\hat{w}_{\alpha}$ of labor value is the variable of use value $\hat{w}_{\alpha}^{*}$, with the domain of definition $G_{\alpha}^{2}$, denoted as

$$
\hat{w}_{\alpha}^{*}=f\left(z_{\alpha}\right)=\hat{w}_{\alpha}\left(b_{\alpha}+i m_{\alpha}\right)+\hat{w}_{\alpha}^{*}\left(b_{\alpha}+i m_{\alpha}\right), z_{\alpha} \in G_{\alpha}^{2}
$$

which shows an economic process that produces the means of subsistence products and creates the use value, that is

$$
f: z_{\alpha}=b_{\alpha}+i m_{\alpha} \rightarrow w_{\alpha}=\hat{w}_{\alpha}+i \hat{w}_{\alpha}^{*}
$$

Obviously, if $w_{\alpha}$ has more than one corresponding for each value of $z_{\alpha}$, then $w_{\alpha}$ is called a multivalued function, including complex polynomials, etc., for example

$$
w_{\alpha}=f\left(z_{\alpha}\right)=x_{\alpha 0} z^{n}+x_{\alpha 1} z^{n-1}+\cdots+x_{\alpha n}
$$

where $w_{\alpha \beta}$ is a complex number of known values and its domain of definition is the entire $z_{\alpha}$ complex plane.

\subsection{Extremum Solutions of the Complex Variable Function of Commodity Value}

The extremum solutions of complex variable function is a complicated problem. There are different conclusions under different conditions that the complex variable functions $f$ of value first-order or second-order derivable defined in the region, compact closed sets, Zero point and singularity. Now, according to the research needs of this paper, we only study problems on extremum solutions of the second-order derivable complex variable function of value in the region or compact closed sets. From $(2-2-2) \sim(2-2-5)$, it is known that a value function $f$ is a complex function defined on an open set $U_{\alpha} \subset G_{\alpha}^{2}$.

If the derivative of $f$ is attained so that all the partial derivatives of $f$ up to order $r$ exist and are continuous, then $f$ is said to be order $r$ differentiable or $f$ is said to be $C^{r}$. Here $r$ can be all positive integers.

If $f$ has continuous partial derivatives of any order, then $f$ is said to be $C^{\infty}$. 
If $f$ has analytic solutions, that is to say, it is expressed as a convergent power series in a neighbourhood of each point on $U_{\alpha}$, then $f$ is to be $C^{\infty}$. In particular, it is easy to prove that the necessary and sufficient condition for the complex variable function $f^{\prime}(z)$ of value defined below to be a holomorphic function is that $f$ has analytic solutions. Also, the necessary and sufficient condition for $f^{\prime \prime}(z)$ to be a harmonic function is that $f$ is holomorphic.

Definition 2.3 Let the complex variable function $f(z)$ of value of any $\alpha$ th commodity be a $r$-order differentiable defined in connected domain $G_{\alpha}^{2}$. Then, for any complex variable function $f: \hat{w}=b+m i \rightarrow \hat{w}^{*}=b+m i$ of commodity value, if it satisfies

$$
f^{\prime}\left(z_{\alpha}\right)=\left\{\begin{array}{l}
\frac{\partial \hat{w}_{\alpha}}{\partial b_{\alpha}}=\frac{\partial \hat{w}_{\alpha}^{*}}{\partial m_{\alpha}} \\
\frac{\partial \hat{w}_{\alpha}^{*}}{\partial b_{\alpha}}=-\frac{\partial \hat{w}_{\alpha}}{\partial m_{\alpha}}
\end{array}\right.
$$

it is called the Cauchy-Riemann equation of commodity value, or holomorphic function for short.

Definition 2.4 Let the complex variable function $f\left(z_{\alpha}\right)$ of value of any $\alpha$ th commodity be a holomorphic function and a second-order differentiable function, and satisfy the following conditions

$$
f^{\prime \prime}\left(z_{\alpha}\right)=\left\{\begin{array}{l}
\frac{\partial^{2} \hat{w}_{\alpha}}{\partial b_{\alpha}^{2}}=\frac{\partial^{2} \hat{w}_{\alpha}}{\partial m_{\alpha} b_{\alpha}} \\
\frac{\partial^{2} \hat{w}_{\alpha}^{*}}{\partial m_{\alpha}^{2}}=-\frac{\partial \hat{w}_{\alpha}^{*}}{\partial b_{\alpha} m_{\alpha}}
\end{array}\right.
$$

Take the derivative of $b$ in (2-3-2), and add the two expressions to be

$$
\Delta \hat{w}_{\alpha}=\frac{\partial^{2} \hat{w}_{\alpha}}{\partial b_{\alpha}^{2}}+\frac{\partial^{2} \hat{w}_{\alpha}}{\partial m_{\alpha}}=0
$$

called as harmonic equation of labor value. Take the derivative of $m$ in (2-3-2), and add the two expressions to be

$$
\Delta \hat{w}_{\alpha}^{*}=\frac{\partial^{2} \hat{w}_{\alpha}^{*}}{\partial b_{\alpha}^{2}}+\frac{\partial^{2} \hat{w}_{\alpha}^{*}}{\partial m_{\alpha}}=0
$$


called as harmonic equation of use value. Obviously, (2-3-3) and (2-3-4) are reversible and equivalent. Therefore, (2-3-2) can be referred to as complex variable function of commodity value.

Definition 2.5 Let $(x)=\left(b_{1}, b_{2}, \cdots, b_{n}, m_{1}, m_{2}, \cdots, m_{n}\right) \in B^{n} \times M^{n} \quad$ be a $2 \mathrm{n}$ dimensional value Euclidean metric space composed of the quantity and quality of $\mathrm{N}$ commodities. Let $G \subset B^{n} \times M^{n}$ be a compact closed set under the natural topology structure of the Euclidean metric space of commodity value. ${ }^{[3]}$ Let $f$ be a bounded value complex variable function defined on $G$, that is $k_{1} \leq f(x) \leq k_{2}, k_{1}, k_{2} \in R$ are the upper and lower bounds of $f(x)$ on $G$ respectively, which are called the boundary points of the effective interval compact set of commodity value complex variable function.

Lemma 2.1 Extremum principle for harmonic function: The maximum and minimum values of a nonconstant function $\mathrm{U}(\mathrm{z})$ that is harmonic in a bounded simply connected domain and continuous on its boundary must occur on the boundary. (Jeffery, 2002, p.780)

Theorem 2.2 (1) If $G$ is a bounded simply connected domain of $B^{n} \times M^{n}$ and $f$ is a harmonic function with constant function properties defined on $G$, then the extremum solutions of $f$ must exist within the inner points of the domain, where when the Hesse matrix is positive definite, the complex variable function of value attains the minimum; when Hesse matrix is negative definite, the complex variable function of value attains the maximum. If the complex variable function $f$ of commodity value is a harmonic function defined on $G$ and, a nonconstant function, then $f$ must attain a maximum or minimum on the boundary of $G$, that is, the equilibrium point of commodity supply and demand.

Proof: Assume that the complex variable function of value of $n$ commodities is a second-order continuously differentiable function $f: G \rightarrow R$ (where $G$ is an open subset in $R^{2 n}$ ), and a Laplace equation, that is, $f$ on $G$ satisfies the equation:

$$
\frac{\partial^{2} f}{\partial x_{1}^{2}}+\frac{\partial^{2} f}{\partial x_{2}^{2}}+\cdots+\frac{\partial^{2} f}{\partial x_{2 n}^{2}}=0
$$

[3] See definition 3.2 below for the detailed description of the Euclidean metric space of commodity value and the corresponding natural topology structure. 
Assume that (2-3-5) is a Hesse matrix, let $\left(x_{\beta}\right)=\left(b_{\alpha}, m_{\alpha}\right) \in B^{n} \times M^{n}, \quad \alpha=1,2, \cdots, n$, $\beta=1,2, \cdots, 2 n$, then when

$$
\frac{\partial^{2} f}{\partial x^{2}}=\left(\begin{array}{l}
\frac{\partial^{2} f}{\partial x_{1}^{2}}, \frac{\partial^{2} f}{\partial x_{1} \partial x_{2}}, \cdots, \frac{\partial^{2} f}{\partial x_{1} \partial x_{2 n}} \\
\frac{\partial^{2} f}{\partial x_{2} \partial x_{1}}, \frac{\partial^{2} f}{\partial x_{2}^{2}}, \cdots, \frac{\partial^{2} f}{\partial x_{2} \partial x_{2 n}} \\
\vdots \\
\frac{\partial^{2} f}{\partial x_{2 n} \partial x_{1}}, \frac{\partial^{2} f}{\partial x_{2 n} \partial x_{2}}, \cdots, \frac{\partial^{2} f}{\partial x_{2 n}^{2}}
\end{array}\right)=0
$$

the second-order partial derivative of the complex variable function of value of $n$ commodities satisfies the condition of (2-3-2), which is a harmonic function. Therefore, at this time $f$ is a constant function defined in the simply connected domain, and there are extremum points. Also, according to the properties of Lagrange multiplier method, when Hesse matrix is positive definite, $f$ attains the minimum; when Hesse matrix is negative definite, $f$ attains the maximum. (Arrow \& Intrigator, 1981, app.B)

(2) If $f$ is not a constant function at this time, it must be an unbounded function. Therefore, $f$ is impossible to attain the maximum or minimum at the inner point of the region. In this case, the extremum solutions of $f$ must be attained on the boundary of the bounded simply connected domain. This can be proved by Lemma 2.1. In particular, according Axiom 6 and Theorem 1 in the new theory of value (Wu, et al., 2020), if the quality of any commodity is constant, there must be an upper bound on the quantity demand of that commodity. In this case, the effective interval $G$ of the definition domain of the complex variable function of commodity value must be a bounded simply connected domain of $B^{n} \times M^{n}$. Therefore, if $f$ is not a constant function at this time, the extremum solutions of $f$ must be attained on the boundary of the bounded simply connected domain. Obviously, the extremum point must be the equilibrium point of commodity supply and demand. Q.E.D.

Economic meaning: The value of a commodity is composed of labor value and use value. At the same time, the labor value and use value of the commodity are determined by the value owned by the quality and quantity. The analysis of the complex variable function of commodity value gives the mathematical theoretical form of the relationship of values of this commodity, and this theorem gives the existence conditions for the maximum or minimum of 
commodity value function, and the relationship between the extremum solutions and the point of supply and demand balance. In the history of economic theory, the first person to analyze the relationship between the labor value and the use value of commodities is Jevons. However, after Jevons, the study of this theory was basically interrupted. From Walras, neoclassical economics began to focus on the analysis of utility function in the field of use value. In this section, we return to Jevons and continue the study of value theory. Note that when the complex variable function of commodity value is a harmonic function, it also has many different properties, such as the mean value theorem of harmonic function. According to the needs of research, these problems are not discussed in this paper. ${ }^{[4]}$

\section{Commodity Value in Real Number Field}

Since Smith, the research on quality in economics is established on the basis of experience. Since the commodities purchased in the market mostly have quality assurance, thus, to simplify the discussion, people can assume that the quality of commodities is constant. In this way, the theory of commodity value can jump away from the complex number field, and discuss it directly in the real number field. At the same time, the content of theoretical research has been greatly simplified.

\subsection{Quantity Vector Space of N Commodities}

Definition 3.1 Let $B^{n}$ be the vector space of an n-dimensional commodity quantities in the real number field $R$,

$$
B^{n}=\left\{b=\left(b_{1}, b_{2}, \cdots, b_{n}\right) \mid b_{\alpha} \in R, 1 \leq \alpha \leq n\right\}
$$

where $B^{n}$ is the set of arrays formed by $n$ ordered commodity quantities $b=\left(b_{1}, b_{2}, \cdots, b_{n}\right) \in B^{n} \quad$ represented by real numbers, and the real number $b_{\alpha}$ is called the $\alpha$ th quantity coordinate of the point. $\forall a, b \in B^{n}, x \in F \quad$ represents an element in a number field, $F$ represents a real number field $R$ and satisfies the following conditions

[4] Along with the analysis of complex variable function of value, we will enter the research category of Ricardo's "a perfect standard or invariable measure of value" (1817, p.32). Here, the theoretical study will involve the concepts of complex variable function, complex Riemannian manifold, Keller manifold, Ricci curvature, hyperbolic space, Hamilton metric, Lorentz inner product, Riemannian symmetric space, fixed point, Chern characteristic class and etc. On the above issues, we will discuss in another paper. 


$$
\left\{\begin{array}{l}
(a+b)_{\alpha}=a_{\alpha}+b_{\alpha} \\
(a x)_{\alpha}=a x_{\alpha}
\end{array}\right.
$$

In this way, the addition and multiplication of commodity quantity vector are defined on $B^{n}$, so that $B^{n}$ becomes an $n$-dimensional value vector space. The $\alpha$ th component $b_{\alpha}$ represents the quantity of the $\alpha$ th commodity in its unit of measurement, and $b_{\alpha}$ can be positive or negative.

In the production field, when $b_{\alpha}$ is positive, it means that the quantity of commodities owned or produced by the producer is $b_{\alpha}$; when $b_{\alpha}$ is negative, it means that the production elements consumed by the producer is the quantity of $b_{\alpha}$.

In the consumption field, when $b_{\alpha}$ is positive, it means that the quantity of commodities purchased by the consumer is $b_{\alpha}$; when $b_{\alpha}$ is negative, it means that the production cost consumed by the consumer is the quantity of $b_{\alpha}$.

\subsection{N-dimensional Linear Normed Space of Commodity Value}

Definition 3.2 Given that the quality of commodities is a constant, and the displacement distance of commodities is determined by uniform acceleration, it can be known from Equation (2-2-1) that $\forall x, y \in B^{n}$ gives the inner product operation of commodity space, let

$$
d(x, y)=\sqrt{\sum_{\alpha=1}^{n}\left(x_{\alpha}+y_{\alpha}\right)^{2}}
$$

Easy to verify, the function $d(x, y)$ satisfies the following three properties: (a) $d(x, y) \geq 0$, and the equal sign holds only when $x=y$; (b) $d(x, y)=d(y, x)$; (c) For any $x, y, z \in B^{n}$, there is inequality $d(x, y)+d(y, z) \geq d(x, z)$. Therefore, $d(x, y)$ is the distance function in $B^{n}$, and the commodity quantity vector space is constructed into an Euclidean metric space $\left(B^{n}, d\right)$. Then there are conclusions: (a) $|\langle x, y\rangle| \leq|x||y|$ (Cauchy inequality); (b) $|x+y| \leq|x|+|y| \quad$ (triangle inequality), where $\langle\bullet\rangle$ represents the inner product operation.

Obviously, we can give $\left(B^{n}, d\right)$ a natural topology structure (Wang, 1978): taking the union of open ball 


$$
B_{x ; r}^{(i)}=\left\{y \in B^{n} \mid d(x, y)<r, i=1,2, \cdots, l\right\}, \quad\left(x \in B^{n}, r>0\right)
$$

as the open set, $B^{n}$ with $d(x, y)$ as the distance function is called the simply connected topology space of commodity value with respect to the production and consumption activities of any $i$ th individual, where $i=1,2, \cdots l$ represents that there are $l$ individuals in the economic system.

Finally, it is known that Cauchy sequences defined at any point in Euclidean metric space $\left(B^{n}, d\right)$ are convergent. (Wang, 1978) Therefore, the Euclidean metric space of commodity value is a complete metric space, that is, Banach linear normed space. Hereinafter, the complete Euclidean metric space $B^{n}$ of commodity value is referred to as commodity value space.

\subsection{Exchange Value of Commodities}

The exchange value of a commodity refers to the proportional relationship between the value owned by the quantity of one commodity and the value owned by the quantity of other commodities. (Ricardo, 1817, p.23) In a more general case, the exchange value of commodities can be expressed as the proportion of the value own by each commodity in unit quantity in the total value of all commodities in the whole society. (Marx, 1867, ch.5) Furthermore, we suppose that the exchange value of any commodity combination is the proportion of the value of the commodity combination to the total value of all commodities. Then, we can give a specific definition of commodity exchange value in this case.

Definition 3.3 Let the rational demand quantity of commodities be $b^{*}=\left(b_{1}^{*}, b_{2}^{*}, \ldots, b_{n}^{*}\right) \in \bar{D} \subset B^{n}$, and $\bar{D}$ be called the subset of rational demand quantity, where $b_{\alpha}^{*} \in \bar{D}_{\alpha}$ represents the rational demand quantity of the $\alpha$ th commodity, and $\bar{D}_{\alpha}$ represents the subset of commodity quantity of the $\alpha$ th component in the vector space of the rational demand quantity of the $\alpha$ th commodity. Therefore, the subset of commodity exchange value under the condition of supply-demand balance is

$$
D=\left\{b \in \bar{D} \subset B^{n} \mid \lambda x+(1-\lambda) y \in \bar{D}, \lambda \in[0,1]\right\}
$$

where $\lambda$ represents the exchange value of any commodity vector $b^{*}$, i.e 


$$
\lambda=\sum_{\alpha=1}^{n} \lambda_{\alpha}=\sum_{\alpha=1}^{n} \frac{\bar{w}_{\alpha} b_{\alpha}^{*}}{\sum_{\alpha=1}^{n} \bar{w}_{\alpha} b_{\alpha}^{*}}
$$

D is called a subset of commodity exchange values.

Theorem 3.1 Subset $D$ of commodity exchange values is a convex set.

Proof: Given that

$$
\sum_{\alpha=1}^{n} b_{\alpha}^{*} \bar{w}_{\alpha}=w
$$

is the total social value of commodities under the condition of supply-demand balance. If the exchange value $\lambda$ of the commodity vector is the weight coefficient of the total commodity value of the whole socio-economic system, that is, from (3-3-2), the exchange value of any $\alpha$ th commodity is

$$
\frac{\bar{w}_{\alpha} b_{\alpha}^{*}}{\sum_{\alpha=1}^{n} \bar{w}_{\alpha}^{*} b_{\alpha}^{*}}=\frac{w_{\alpha}}{w}=\lambda_{\alpha}
$$

Then, let $w=1$, we have

$$
\lambda_{\alpha}=\frac{w_{\alpha}}{1} \Rightarrow w_{\alpha} \in[0,1] \Rightarrow \sum_{\alpha-1}^{n} w_{\alpha}=1 \Rightarrow \sum_{\alpha-1}^{n} \lambda_{\alpha}=1
$$

Thus, for any two points $x, y \in D^{n}, \omega \in[0,1]$, there must be expressions

$$
\begin{aligned}
& x=\lambda_{1} x^{(1)}+\cdots+\lambda_{m} x^{(m)}, \text { where } \sum_{\alpha=1}^{m} \lambda_{\alpha}=1 \\
& y=\mu_{1} y^{(1)}+\cdots+\mu_{n} y^{(n)}, \text { where } \sum_{\beta=1}^{n} \mu_{\beta}=1
\end{aligned}
$$

so

$$
\omega x+(1-\omega) y=\omega \lambda_{1} x^{(1)}+\cdots+\omega \lambda_{m} x^{(m)}+(1-\omega) \mu_{1} y^{(1)}+\cdots+(1-\omega) \mu_{n} y^{(n)}
$$

also

$$
\begin{gathered}
\omega \lambda_{1}+\cdots+\omega \lambda_{m}+(1-\omega) \mu_{1}+\cdots+(1-\omega) \mu_{n} \\
\omega\left(\lambda_{1}+\cdots+\lambda_{m}\right)+(1-\omega)\left(\mu_{1}+\cdots+\mu_{n}\right)
\end{gathered}
$$




$$
=\omega+(1-\omega)=1
$$

Since $\omega \in[0,1]$, there must be $\omega x+(1-\omega) y \in D$. This shows that $D$ is a convex set. Q.E.D.

Economic meaning: The qualitative concept of exchange value was first given by Ricardo, which indicates the ratio of the value of unit quantity of one commodity to the value of unit quantity of any other commodity. Here, we give the mathematical definition of exchange value and analyze the convex properties of exchange value. It should be noted that on the surface, the convexity of the exchange value of commodities defined in this paper is the same as that of the indifference utility curve in the general equilibrium theory. However, in fact, there are essential differences between the two. The former is the upper graph of the convex function of commodity market price, that is, the commodity value and price in equilibrium. The latter is the convex function of commodity market price itself, that is, the general theoretical form of commodity price in a non-equilibrium state. For the detailed analysis of the above conclusions, see Sections 4 and 5 of this paper.

\subsection{Fixed Point of Self Mapping of Commodity Value Space}

Definition 3.4 Assuming that $\forall x, y \in L$, the exchange value of commodities is expressed by the total value coefficient, that is $\forall \lambda_{1}, \lambda_{2} \in W$, if there is $\lambda_{1} x+\lambda_{2} y \in L$, then $L \subset B^{n}$ is called the value subspace of $B^{n}$. If $L=\left\{x_{1}, x_{2}, \cdots, x_{p}\right\}$, then $x=\lambda_{1} x_{1}+\lambda_{2} x_{2}+\cdots+\lambda_{p} x_{p}$ is called the value linear combination of $x_{1}, x_{2}, \cdots, x_{p}$.

Definition 3.5 $\forall b \in B^{n}, b^{*} \in D \subset B^{n}$, defines the distance $d(b, D)$ between $b$ and $D$ as: $d(b, D)=\inf \left\{\left|b-b^{*}\right| \mid b^{*} \in D\right\}$, which is called the distance function of subset of rational demand quantity in $B^{n}$ induced by value Euclidean metric $d(x, y)$.

Definition 3.6 Suppose $T: D \rightarrow D$ is a self mapping of subset of rational demand quantity for commodities, if there exists a commodity value real number $\lambda \in W, 0<\lambda<1$, so that for all $\forall x, y \in D \subset B^{n}$, for Euclidean metric function $d(x, y)$ holds,

$$
d(T x, T y) \leq \lambda d(x, y)
$$

then $T$ is called as a compression mapping of commodity value. 
Lemma 3.1 (Compression mapping theorem) Let $X$ be a complete metric space and $T$ be a compression mapping on $X$, then $T$ has one and only one fixed point. (Banach, 1922)

Theorem 3.2 Let $C(D)$ be the set distance functions $d(b, D)$ of a subset of rational demand quantity defined in $B^{n}$, then the commodity value self-mapping $T: D \rightarrow D$ must have a unique fixed point.

Proof: (1) According to Theorem 3.1, $B^{n}$ is a Euclidean metric space. Therefore, $B^{n}$ is complete. Assuming that $D$ is a subset of $B^{n}$, it can be known from the property that the value of $\forall x \in D$ is defined by the Euclidean metric $f(x)=\sqrt{x x}=|w|$ that $\forall x, y \in D$, $\forall \lambda_{1}, \lambda_{2} \in W \cong R$, all have $\lambda_{1} x+\lambda_{2} y \in D$ such that $D \cong L \subset B^{n}$ is a value subspace of $B^{n}$. Therefore, $D \subset B^{n}$ is also a complete metric space.

(2) According to Property (c) of inner product operation, for any $x, y, z \in B^{n}$, there is inequality $d(x, y)+d(y, z) \geq d(x, z)$. Therefore, in the case of $D \subset B^{n}$, there must be $D \cong \tilde{D} \subset B^{n}$, that is, there must exist a commodity value real number $\lambda \in W, 0<\lambda<1$, so that for all $\forall x, y \in D \subset B^{n}$, for Euclidean metric function $d(x, y)$ holds,

$$
d(T x, T y) \leq \lambda d(x, y)
$$

thus $T$ is a compression mapping of commodity value.

(3) According to Lemma 3.1, $T$ has one and only one fixed point. Specifically, take any point $b_{0} \in D \subset B^{n}$ and start with $b_{0}$ for the iterative procedure: let

$$
\begin{aligned}
& b_{1}=T b_{0}, \\
& b_{2}=T b_{1}=T^{2} b_{0}, \\
& \cdots \cdots \\
& b_{n}=T b_{n-1}=T^{n} b_{0},
\end{aligned}
$$

According to the Euclidean metric properties of value space $B^{n}$, we know that $\left\{b_{n}\right\}$ is a basic sequence in $D^{n}$. According to the above theorem proof (2), $T$ has compressibility, i.e

$$
\begin{aligned}
& d\left(b_{1}, b_{2}\right)=d\left(T b_{0}, T b_{1}\right) \leq \lambda d\left(b_{0}, b_{1}\right)=\lambda d\left(b_{0}, T b_{0}\right) \\
& d\left(b_{2}, b_{3}\right)=d\left(T b_{1}, T b_{2}\right) \leq \lambda d\left(b_{1}, b_{2}\right) \leq \lambda^{2} d\left(b_{0}, T b_{0}\right)
\end{aligned}
$$

It can be proved by mathematical induction 


$$
d\left(b_{n}, b_{n+1}\right) \leq \lambda^{n} d\left(b_{0}, T b_{0}\right), \quad n=1,2, \cdots
$$

thus

$$
\begin{aligned}
d\left(b_{n}, b_{n+p}\right) & \leq d\left(b_{n}, b_{n+1}\right)+d\left(b_{n+1}, b_{n+2}\right)+\cdots+d\left(b_{n+p-1}, b_{n+p}\right) \\
& \leq\left(\lambda^{n}+\lambda^{n+1}+\cdots+\lambda^{n+p-1}\right) d\left(b_{0}, T b_{0}\right) \\
& =\frac{\lambda^{n}\left(1-\lambda^{p}\right)}{1-\lambda} d\left(b_{0}, T b_{0}\right) \\
& \leq \frac{\lambda^{n}}{1-\lambda} d\left(b_{0}, T b_{0}\right)
\end{aligned}
$$

Since the exchange value $0<\lambda<1$, we know that $\left\{b_{n}\right\}$ is the basic sequence.

Since $B^{n}$ is complete, there must be $b^{*} \in B^{n}$, make $b_{n} \rightarrow b^{*}(n \rightarrow \infty)$, and then from the continuity of the contraction operator, let $n \rightarrow \infty$ take the limit in $b_{n+1}=T b_{n}$, we get

$$
b^{*}=T b^{*}
$$

that is, $b^{*}$ is the fixed point of $T$.

Prove the uniqueness of fixed point. Suppose $\bar{b}^{*}$ is also a fixed point of $T$, that is $T \bar{b}^{*}=\bar{b}^{*}$, then

$$
\rho\left(b^{*}, \bar{b}^{*}\right)=d\left(T b^{*}, T \bar{b}^{*}\right) \leq \lambda d\left(b^{*}, \bar{b}^{*}\right)
$$

Since $0<\lambda<1$, if (3-4-2) is to be established, according to the nature of the function $d(x, y)$, there must be $d\left(b^{*}, \bar{b}^{*}\right)=0$, that is, $b^{*}=\bar{b}^{*}$. Q.E.D.

Economic meaning: This theorem indicates that, assuming that the social production process is a social reproduction process with constant quality, there must exist fixed points under the value self-mapping in each economic circulation process. Specifically, in the period of barter commodity economy, as long as the social reproduction process is a reproduction process with constant quality, meanwhile the premise condition given in this theorem is satisfied, then the market supply and demand relationship will be in a state of balance, and the value of all commodities would be stable and unchanged. In this case, as long as all people follow the principle of equivalent exchange when they exchange commodities in the market, the final result of Commodity Exchange will inevitably tend to the exchange value owned by each commodity under the spontaneous market regulation mechanism. Here, the theorem that there must be a single fixed point for the self-mapping of Commodity Exchange value is the mathematical theoretical expression of the above objective economic law - Smith's invisible 
hand and Marx's law of value - in the period of barter without the money medium and the price adjustment.

\section{Equilibrium Price of Commodities}

The real socio-economic system is the monetary economic system. Here, the value of commodities is realized through monetary media and price. In particular, in an ideal commodity economic system, if the value of commodities is known, there is a linear transformation relationship between the prices of various commodities and the value of the currency of commodities - precious metal currency - on the premise of equivalent exchange. ${ }^{[5]}$

\subsection{Affine Mapping of Commodity Prices}

Definition 4.1 Let the currency subset $H \subset R$ be a finite quantitative set of precious metal currencies - such as gold, and a bounded closed set of one-dimensional real numbers, representing the commodity that acts as the universal equivalent among $\mathrm{n}$ commodities. It is assumed that all other non-gold commodities of $n$ commodities are equivalents which are all expressed by gold as the measure of value, then we can define a price affine mapping satisfying the exchange value constraint in commodity quantity space $B_{+}^{n}{ }^{[6]}$ as follows

$$
\begin{gathered}
f_{p}^{n-1}: D \subset B_{+}^{n} \rightarrow P^{n-1} \\
\left(b_{1}, b_{2}, \cdots b_{n-1}, h\right) \mapsto\left(p_{1}, p_{2}, \cdots p_{n-1}\right)
\end{gathered}
$$

Here, the price of the $\alpha$ th commodity can be expressed as

$$
p_{\alpha}=\left(b_{\alpha}^{*} \lambda_{\alpha}\right)=p\left(b_{\alpha}, h\right)=\frac{\frac{\bar{w}_{\alpha} b_{\alpha}^{*}}{\sum_{\alpha=1}^{n-1} \bar{w}_{\alpha} b_{\alpha}^{*}}}{b_{\alpha}^{*}}=\frac{\lambda_{\alpha} h}{b_{\alpha}^{*}}=\bar{\lambda}_{\alpha} h
$$

where $\alpha=1,2, \ldots, n-1, \quad p_{\alpha}$ is called the equilibrium price of the commodity, $\bar{w}_{\alpha}$ is the unit

[5] In this section, we only examine the commodity price problem in the field of labor value, that is, assuming the case that the quality of commodities is constant. So the quantity space of commodities belongs to the real number field, which can be positive or negative, where the positive corresponding to the labor value, is the product of the production field, and the negative corresponding to the use value, is the product of the consumption field. The price of the corresponding commodity can also be positive or negative. Positive prices are incomes and negative prices are expenditures.

[6] In this paper, to simplify the discussion, we will only look at commodity prices for which precious metal money acts as an equivalent. So here, money itself has value. 
value of the commodity, $\bar{\lambda}$ is the exchange value of the commodity in the $\alpha$ th industry, $\bar{\lambda}_{\alpha}$ is the exchange value of the unit quantity of the commodity in the $\alpha$ th industry, and $b_{\alpha}^{*} \in[0,+\infty]$ is the rational demand quantity of each commodity, which is a real number in the closed range of real numbers greater than zero to positive infinity. Thus, $P^{n-1}$ is called the equilibrium price space of commodities, and $p \in P^{n-1}$ is called the equilibrium price vector of $n-1$ commodities.

In particular, the equilibrium price of the same commodity includes positive and negative values. The positive price corresponds to the labor value in the production field, and the negative price corresponds to the use value in the consumption field. In real life, the specific manifestation is the commodity price corresponding to the assets and liabilities in the balance sheet.

\subsection{Relationship and Property between the Value and Price of Commodities}

Definition 4.1 gives the mathematical theoretical form of the conversion of commodity value into price under the gold standard system. Here, the value of commodities metamorphoses into price. Therefore, today's commodities are no longer personal wealth active in the market trade of small-scale peasant economies, but huge social wealth with unified measure of value in the legal currency circulation field of various countries.

In this case, the economic relationship between the value and price of commodities has rich mathematical representations:

Definition 4.2 (1) Set the quantity subset of any commodity $L \subset B^{n}$. If for $\forall x, y \in L$, in (4-1-2) there always is exchange value $\lambda \in R$, so that the relation of $1-\lambda x+\lambda y \in L$ exists, then $L$ is called the affine set of $B_{+}^{n}$. Obviously, all affine sets are convex. (Rockefeller, 1970, p.10)

(2) Suppose that the subset $\left\{x_{1}, x_{2}, \cdots, x_{p}\right\} \subset B^{n}$ composed of multiple commodity vectors under the exchange value relationship is a $B^{n}$ vector group. If the social exchange value of commodities is $\lambda_{1}, \lambda_{2}, \cdots, \lambda_{p} \in R, \quad$ so that $\lambda_{1} x_{1}+\lambda_{2} x_{2}+\cdots+\lambda_{p} x_{p}=w$, the vector group of a commodity is a linear combination of equilibrium prices.

(3) Suppose that $L: \lambda_{1} L+\cdots+\lambda_{n} L, \forall \lambda_{1} \cdots, \lambda_{n} \in R, L$ is called equilibrium price affine 
subspace of $B^{n}$.

(4) Suppose that $K: \lambda_{1} K+\cdots+\lambda_{n} K \subset K, \forall \lambda_{1} \cdots, \lambda_{n} \in[0,1], \sum_{\alpha=1}^{n} \lambda_{\alpha}=1, \quad K \quad$ is equilibrium price convex set of $B^{n}$.

(5) Suppose that $C: \lambda_{1} C+\cdots+\lambda_{n} C \subset C, \forall \lambda_{1} \cdots, \lambda_{n}>0, C$ is equilibrium price convex cone of $B^{n}$.

$n$ of (1) - (5) above is any integer $\geq 2$.

(6) The set $E \subset B^{n}$ is an equilibrium price convex hull, if it satisfies the following condition:

$$
E:=\bigcap_{X \subseteq D \subseteq B^{n}} K
$$

where $X$ is the true subset of equilibrium price space $P^{n-1}$, the convex hull of $X$ is constructed from a linear combination of all the equilibrium price combination points $\left(p_{1}, p_{2}, \cdots, p_{n}\right)$ in $X$, that is

$$
\operatorname{co} E:=\left\{\sum_{i=1}^{l} p_{i} b_{i} \mid b_{i} \in X, \sum_{\alpha=1}^{n-1} p_{\alpha}=\sum_{\alpha=1}^{n-1} \lambda_{\alpha}=\frac{\bar{w}_{\alpha} b_{\alpha}^{*}}{\sum_{\alpha=1}^{n-1} \bar{w}_{\alpha} b_{\alpha}^{*}} h=1\right\}
$$

Definition 4.3 If the mapping $T$ from the affine set $M$ in $B^{n}$ to $P^{n-1}$ maintains the affine combination, i.e

$$
T\left(\sum_{\alpha=1}^{p} \lambda_{\alpha} x_{\alpha}\right)=\sum_{\alpha=1}^{p} \lambda_{\alpha} T\left(x_{\alpha}\right)
$$

where $\sum_{\alpha=1}^{p} \lambda_{\alpha}=1, \lambda_{\alpha} \in R, \alpha=1,2, \ldots, p$, then $T$ is an affine transformation. Therefore, it is easy to see from (4-1-2) that there is $T x=A x+p$, where $A=\left(y_{\alpha \beta}\right)_{n \times(n-1)}$ is a coefficient matrix defined by $f_{p}$, and $y \in B^{n} \times P^{n-1}, \quad p \in P^{n-1}$.

Definition 4.4 Let $C \subset B_{+}^{n}, x \in C$, if there is an $\varepsilon>0$ such that $x+\varepsilon B \subset C$, then $x$ is the interior point of $C$. The set of all the interior points of $C$ is called the interior of $C$ 
and can be expressed as int $C=\{x \mid \exists x+\varepsilon B \subset C\}$. The set of all interior points of $C$ is called the closure of equilibrium price convex cone, denoted by $\mathrm{clC}$.

Definition 4.5 Let $C \subset B^{n}$, if there is a point column $\left\{x_{k}\right\}, x_{k} \in C, k=1,2, \cdots, n \cdots$ that satisfies $\lim _{k \rightarrow \infty} x_{k}=x$, then $x$ is the limit point of $\left\{x_{k}\right\}$, and $f(x)$ is a continuous function.

Definition 4.6 Let $P^{n-1}$ be a real linear space and has an ordered structure, that is, for some vector pairs $(x, y)$ in $P^{n-1}, x \geq y$ (or $x \leq y$ ), " $\geq$ " satisfies the following conditions: for any $x, y, z \in B_{+}^{n}$ and exchange value real number $\lambda$,

(1) $x \geq x$; if $x \geq y$ and $x \leq y$, then $x=y$; if $x \geq y$ and $y \geq z$, then $x \geq z$.

(2) if $x \geq y$, then $x+z \geq y+z$.

(3) if $x \geq y, \lambda \geq 0$, then $\lambda x \geq \lambda y$.

thus $P^{n-1}$ is called ordered linear space.

Theorem 4.1 (1) In the process of commodity exchange, for all $n-1$ commodities, if both sides of the exchange conduct equivalent exchange according to the exchange value, and the quantity of commodities keeps the balance between supply and demand, then the price space of commodities $P^{n-1}$ is a hyperplane in $B^{n}$ and also a convex set; (2) In the range from $n-1$ kinds to one kind of commodity, any linear combination of multiple commodity prices can be represented as a mapping like $T b=A b+a$ under the condition of affine transformation, where $A$ is the linear transformation from $B^{n}$ to $P^{m}, b \in B^{n}, a \in P^{m}$, $1 \leq m<n, \quad$ so that $P^{n-1}=\left\{x \in B^{n} \mid x=(p, h), p y \leq w\right\}, \quad y=\left(b_{1}, b_{2}, \cdots, b_{n-1}\right) \in R^{n-1}, \quad h \in H \quad$, $R^{n-1} \times H=B^{n} ;(3) \quad P^{n-1}$ is ordered linear space

Proof: (1) Let $p \in P^{n-1}, A$ is $n \times(n-1)$ ordered matrix, then the set

$$
P^{n-1}=\left\{x \in B^{n} \mid A x=p\right\}
$$

According to equation (3-3-2) of commodity exchange value, equation (4-1-2) shows that the equilibrium price of a commodity is equal to the exchange value per unit of commodity multiplied by the aggregate amount of monetary values, i.e.

$$
p=\left(p_{1}, p_{2}, \cdots p_{n-1}\right)=h\left(\bar{\lambda}_{1} b_{1}, \bar{\lambda}_{2} b_{2}, \cdots, \bar{\lambda}_{n-1} b_{n-1}\right)=h\left(\bar{w}_{1} b_{1}, \bar{w}_{2} b_{2}, \cdots, \bar{w}_{n-1} b_{n-1}\right) \in P^{n-1}
$$


is a Hyperplane set in $B_{+}^{n}$. Since $\forall x, y \in P^{n-1}, \lambda \in R$, when $z=(1-\lambda) x+\lambda y$, there is $A z=(1-\lambda) A x+\lambda A y=(1-\lambda) p+\lambda p=p, \quad z \in P^{n-1}$. Thus, according to Definition 4.3, it is a Hyperplane.

In addition, since $P^{n-1}$ is a hyperplane, given by Definition 4.3 that $\forall x, y \in P^{n-1}, \lambda \in R$, have $1-\lambda x+\lambda y \in P^{n-1}$. Therefore, $P^{n-1}$ is a convex set.

(2) Given $P^{n-1}=\left\{x \in B_{+}^{n} \mid x=(p, h), p y \leq w\right\}$ that the equilibrium price space of commodities is composed of all elements that the sum of the product of price vector and commodity quantity vector is less than and equal to the total social value of commodities. Therefore, it is a set satisfying linear constraints. According to (4-1-2), let $T=f_{p}$ is a affine transformation, let $a=T \theta, A x=T x-a$, easy to verify that $A$ is a linear transformation from $R^{n}$ to $R^{m}$. On the contrary, let $A$ be a linear transformation from $R^{n}$ to $R^{m}$ and have $T x=A x+a$, so for $\forall x, y \in R^{n}, \lambda \in R$, the condition of (4-2-1) is satisfied. So $T=f_{p}$ is an affine transformation. At the same time, because $y=\left(b_{1}, b_{2}, \cdots, b_{n-1}\right) \in R_{+}^{n-1}, R_{+}^{n-1} \times H=B_{+}^{n}$, for $P^{n-1}=\left\{x \in B_{+}^{n} \mid x=(p, h), p y=w\right\}$, must meet the condition of (4-2-1). Therefore, the conclusion (2) of this theorem is valid.

(3) Given the commodity value space $B^{n}$ is an Euclidean metric space. Therefore, under Euclidean metric, $B^{n}$ is an ordered linear space. At the same time, it is also known that $P^{n-1}$ is an affine set, that is, $\forall x, y \in P^{n-1}, \lambda \in R$, always have $1-\lambda x+\lambda y \in P^{n-1}$. Therefore, set the quantity subset of any commodity $L \subset B_{+}^{n}$. If for $\forall x, y \in L$, there is exchange value $\lambda \in R$ in (4-1-2) so that the relationship of $1-\lambda x+\lambda y \in L$ exists, then $L$ is called the affine set of $B_{+}^{n}$. Therefore, there is $L: \lambda_{1} L+\cdots+\lambda_{n} L, \forall \lambda_{1} \cdots, \lambda_{n} \in R$ naturally, so that $L$ is the equilibrium price affine subspace of $B_{+}^{n}$. So, $P^{n-1}$ is also an ordered linear space. Q.E.D.

Economic meaning: This theorem shows that in a closed economic system, if the supply and demand of all commodities are in equilibrium, and the principle of equivalent exchange is realized in all economic activities, then the value of all commodities will be under affine transformation, making its price behave as the n-1-dimensional hyperplane of the n-dimensional commodity value space. Generally speaking, the value of all commodities will 
be allocated to the currency of unit quantity in proportion. In this case, people can calculate relatively accurately through the conversion between the quantity and price of commodities and the currency. Obviously, this is completely different from the period of barter when people can only estimate the value of commodities by feeling. However, we must be soberly aware that the existence of the above pure and ideal environment is conditional, that is, we must ensure that the principle of market supply and demand balance and equivalent exchange can be completely realized.

\section{Market Price Under Non-equilibrium State}

The market price of commodities, in a complex external environment - commodity output may deviate from rational demand quantity. This form of price is the most common form of economy - is the manifestation of commodity equilibrium price. Specifically, in any commodity production system under pure conditions, if the market supply of any commodity quantity is variable and the demand elasticity of the commodity is zero, ${ }^{[7]}$ under the adjustment mechanism of the market price function, when the commodity is in short supply, the market price of the commodity is higher than the equilibrium price; On the contrary, in the case of oversupply, the market price of commodities is lower than the equilibrium price. (Walras, 1900, preface to the fourth edion, p.40-41) Therefore, economics needs to focus on the price problem under the unbalanced state of supply and demand.

\subsection{Nature of the Market Price of Commodities}

When the quality of commodities is constant, the market price of commodities has two types and four different forms: (1-1) the market price of means of subsistence products in the form of labor value; (1-2) market price of means of subsistence products in the form of use value; (2-1) market price of means of production products in the form of labor value; (2-2) market price of means of production products in the form of use value. In order to facilitate understanding, we first investigate the relationship of (1-1) and (1-2), which is a inverse relationship symmetric on the horizontal axis. After understanding the relationship of (1-1) and (1-2), (2-1) and (2-2) are easy to understand, the discussion can be neglected. In the

[7] If there is the phenomenon of non-zero demand elasticity of commodities, it indicates that the price functions of different commodities might be non-linearly correlated. In this case, the price function of the commodity is complicated, which we will not discuss in this paper. However, in spite of this, the theoretical conclusion given in this paper is still applicable to explain the general economic laws existing in the phenomenon of non-linear correlation. 
second part of this section, we will analyze the market price of two types and four different forms above. There, the theoretical analysis will be carried out in the concave-convex function and the corresponding image on a hyperboloid.

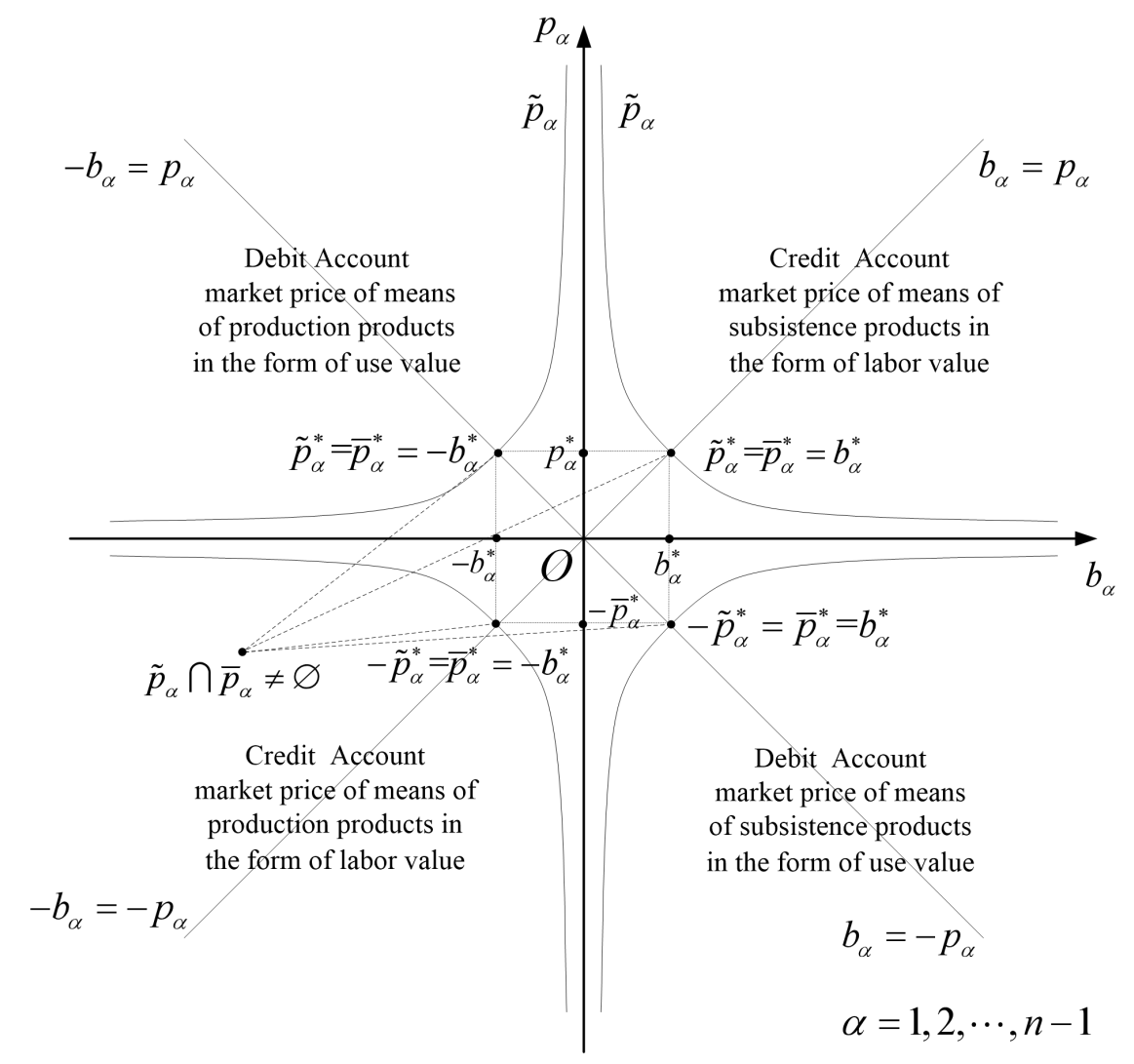

\section{Diagram of Commodity Market Price}

Definition 5.1 Let $B^{n}=B^{n-1} \times H$ indicate that $B^{n}$ is the Cartesian product of the commodity space $B^{n-1}$ as the physical carrier of commodity value and the commodity set $H \cong R \quad$ as money. Let $x=\left(b_{1}, b_{2}, \cdots, b_{n}\right) \in U \subset B^{n-1}$ be the subset of the actual supply quantity of commodities; $x^{*}=\left(b_{1}^{*}, b_{2}^{*}, \cdots, b_{n-1}^{*}\right) \in S \subset B^{n-1}$ be n-1 subsets of the rational demand quantity, which is known exogenous constants; $\quad p=\left(p_{1}, p_{2}, \cdots, p_{n-1}\right) \in V \subset P^{n-1}$ be n-1 subsets of the equilibrium price, which is known exogenous constants; and $F$ be a concave-convex function on hyperbola of market price mapped from $U$ to $[-\infty,+\infty]$.

Further, let the commodity value space $B_{+}^{n-1}$ of means of subsistence corresponding to labor value be a positive half space, that is, $B_{+}^{n-1}$ is the Euclidean measurement space that reduces the positive half commodity value of precious metal commodities acting as money, or $B_{+}^{n-1}$ is 
a set composed of $n-1$ commodity quantity vectors greater than or equal to zero.

Note that $U_{+} \subset B_{+}^{n-1}$ is a subset of the actual output of n- 1 commodities.

Let $x_{+} \in U_{+}$, then the market price function family of commodities in positive half space can be expressed as

$$
\begin{gathered}
F\left(x_{+}\right)=\tilde{p}_{+} \\
\left(b_{1}, b_{2}, \cdots, b_{n-1}\right)_{+} \rightarrow\left(\tilde{p}_{1}, \tilde{p}_{2}, \cdots, \tilde{p}_{n-1}\right)_{+}
\end{gathered}
$$

where the market price function of the positive half space of the means of subsistence commodities in the form of labor value is $f_{\tilde{p}}^{(\alpha)}\left(x_{+}\right)=\tilde{p}_{\alpha_{+}}$. Then the family of sets $\left\{f_{\tilde{p}}^{(\alpha)}\right\}_{+}$ of market price functions in positive half space of means of subsistence commodities is composed of $n-1$ concave functions, denoted as

$$
\begin{aligned}
f_{\tilde{p}}^{(\alpha)}: U_{+} & \rightarrow \tilde{P}_{\alpha+} \cong R \cup\{+\infty\} \\
\left(x_{+}\right) & \mapsto \tilde{p}_{\alpha+}
\end{aligned} \quad, \quad \alpha=1,2, \cdots, n-1
$$

Specifically, the market price of a means of subsistence commodity in the form of labor value is composed of the product of the supply-demand balance coefficient and the equilibrium price of the commodity, that is the market price of the $\alpha$ th commodity is

$$
f_{\tilde{p}}^{(\alpha)}\left(x_{+}\right)=\frac{b_{\alpha+}^{*}}{b_{\alpha+}} \frac{\bar{w}_{\alpha+}}{\sum_{\alpha=1}^{n-1} \bar{w}_{\alpha+} b_{\alpha+}^{*}} h=\frac{b_{\alpha+}^{*}}{b_{\alpha+}} p_{\alpha+}=\tilde{p}_{\alpha+}
$$

where $\alpha=1,2, \cdots, n-1, b_{\alpha_{+}} \in[0,+\infty]$ and $b_{\alpha^{+}}^{*} \in[0,+\infty]$ are the possible variation ranges of the actual supply quantity and rational demand quantity of the $\alpha$ th commodity respectively. $\tilde{p}_{\alpha^{+}} \in[0,+\infty]$ is the change range of the market price under different supply and demand relations. Thus, $\forall \tilde{p}_{+} \in \tilde{P}_{+}^{n-1}$ is the element in the commodity market price set composed of the price vectors of $n-1$ commodities, and $\tilde{P}_{+}^{n-1}$ is called the family of sets of commodity market prices.

Definition 5.2 Given that $f_{\tilde{p}}^{(\alpha)}: U_{+} \rightarrow \tilde{P}_{\alpha+}$ is a real valued function on $U_{+}$, let $y_{+} \in U_{+}$

$$
\operatorname{epi} f_{\widetilde{p}}^{(\alpha)}=\left\{y_{+}=\left(b_{+}, b_{+}^{*}\right) \in U_{+} \times S_{+} \times P_{+}^{n-1} \mid x_{+} f_{\widetilde{p}}^{\alpha}\left(y_{+}\right) \leq \omega\right\}
$$

is called the subset of the upper graph of $f_{\tilde{p}}^{(\alpha)}\left(y_{+}\right)$, which represents the set composed of the 
market price corresponding to the labor value in the production field and the corresponding commodity quantity vectors.

Lemma $5.1 f_{\tilde{p}}^{(\alpha)}$ is a convex function on a convex set $D \subset P^{n-1}$ if and only if the upper graph $\operatorname{epi} f_{\tilde{p}}^{\alpha}$ of $f_{\tilde{p}}^{(\alpha)}$ is a convex set of $B^{n-1} \times H=B^{n}$. (Rockefeller, 1970, p.19-25)

Definition 5.3 If a function of a commodity market price corresponding to labor value is a convex set, then when $f_{\tilde{p}}^{(\alpha)}(b) \mapsto\left\langle b^{*}, b\right\rangle \in R$ satisfies the rational demand quantity $b^{*} \in D_{+} \subset B_{+}^{n}$, and the actual supply quantity $b \in B_{+}^{n}, \lambda, \mu \in R$, there always is $\left\langle b^{*} \mid \lambda b^{*}+\mu b\right\rangle=\lambda\left\langle b^{*}, b\right\rangle+\mu\left\langle b^{*}, b\right\rangle$, it is called the linear form of market price in the upper half space of the rational demand quantity vector of $b^{*}$ on $B_{+}^{n}$.

Theorem 5.1 (1) The market price $f_{\tilde{p}}^{(\alpha)}$ of means of subsistence commodities in the form of labor value is a concave function; (2) The upper graph of the market price function $f_{\tilde{p}}^{(\alpha)}$ of means of subsistence commodities in the form of labor value is a convex set; (3) Let the aggregate amount of market price of any $\alpha$ th commodity of means of subsistence in the form of labor value be the actual supply quantity multiplied by the market price, that is $b_{\alpha+} \tilde{p}_{\alpha}=\omega_{\alpha}$, let the aggregate amount of equilibrium price of any $\alpha$ th commodity of means of subsistence in the form of labor value be the rational demand quantity multiplied by the equilibrium price, that is $b_{\alpha+}^{*} p_{\alpha}=\varpi_{\alpha}$, the aggregate amount of market price is constantly equal to the aggregate amount of equilibrium price of any $\alpha$ th commodity of means of subsistence in the form of labor value, that is $\omega_{\alpha}=\varpi_{\alpha} \cdot{ }^{[8]}$

Proof: (1) According to Theorem 3.1, the value space of commodities is Euclidean metric space. Therefore, the convex function defined on $B^{n}$ is Euclidean norm, that is

$$
|b|=\langle b, b\rangle^{1 / 2}=\left(z_{1}^{2}+z_{2}^{2}+\cdots+z_{n}^{2}\right)^{1 / 2}
$$

Obviously, the convex property of (5-1-5) can be given by triangular inequality, that is, for $\lambda \geq 0$

[8] From a purely mathematical point of view, the function of the aggregate amount of commodity price is exactly the same as Hicks's indifference curve. However, the economic meaning of this theorem (3) is completely different from that of Hicks indifference curve. Here, economic theory gives two different explanations to the same economic phenomenon. But there is only one truth. 


$$
\left|b_{1}+b_{2}\right| \leq\left|b_{1}\right|+\left|b_{2}\right|,|\lambda b|=\lambda|b|
$$

holds. According to theorem 4.1, the price mapping of commodities is the hyperplane of the commodity value space under the exchange value affine mapping. Therefore $\forall x, y \in B^{n}$, $\lambda, \mu \in R$, when $f_{\tilde{p}}^{(\alpha)}(x) \mapsto\left\langle x^{*}, x\right\rangle \in R$, the conditions of $\left\langle x^{*}, \lambda x+\mu y\right\rangle=\lambda\left\langle x^{*}, x\right\rangle+\mu\left\langle x^{*}, x\right\rangle$ is satisfied. Therefore, the market price function of commodities is a convex or concave function.

Specifically, in the process of commodity exchange, for all $n-1$ kinds of means of subsistence commodities in the form of labor value, if both sides of the exchange conduct equivalent exchange according to the exchange value, and the quantity of commodities maintains the balance between supply and demand, it can be seen from Theorem 4.1 (1) that the price of any $\alpha$ th commodity of means of subsistence in the form of labor value is the equilibrium price. If both sides of the exchange conduct equivalent exchange according to the exchange value, and the quantity supply of commodities cannot maintain the balance between supply and demand, it can be seen from definition 5.1 and formula (5-1-3), the market price of any $\alpha$ th commodity of means of subsistence in the form of labor value is an inverse proportional function of the equilibrium price, ${ }^{[9]}$ that is

$$
\tilde{p}_{\alpha+}=\frac{b_{\alpha+}^{*}}{b_{\alpha+}} p_{\alpha+}
$$

refers to the market price of means of subsistence commodities in the form of labor value in the production field, that is, the market price corresponding to the labor value.

Further, let the equilibrium price of any $\alpha$ th commodity of means of subsistence in the form of labor value be 1 , then the commodity market price is a piecewise function

$$
\left\{\begin{array}{ll}
f_{\tilde{p}}^{(\alpha)}\left(x_{+}\right)=\tilde{p}_{\alpha+}<1, & \text { 当 } b_{\alpha+}^{*}>b_{\alpha+} \\
f_{\tilde{p}}^{(\alpha)}\left(x_{+}\right)=\tilde{p}_{\alpha+}=1, & \text { 当 } b_{\alpha+}^{*}=b_{\alpha+} \\
f_{\tilde{p}}^{(\alpha)}\left(x_{+}\right)=\tilde{p}_{\alpha+}>1, & \text { 当 } b_{\alpha+}^{*}<b_{\alpha+}
\end{array} \quad \alpha=1,2, \cdots, n-1\right.
$$

Therefore, the market price function of means of subsistence commodities in the form of labor value is a concave function with $b_{\alpha+}^{*}$ as the discontinuity. As for $b_{\alpha_{+}} \in[0,+\infty]$,

[9] According to the law of diminishing marginal utility, Walras (1874, p.86-88) deduced that the prices of two commodities in the state of equivalent exchange have an inverse proportional relationship. However, Walras did not realize that the inverse proportional relationship of commodity market prices would also exist in the unbalanced state of supply and demand. 
$\tilde{p}_{\alpha+} \in[0,+\infty]$, when $b_{\alpha}$ does not reach the equilibrium point $b_{\alpha+}^{*}$ of supply and demand, the supply of means of subsistence commodities is in short supply, and the market price $\tilde{p}_{\alpha}$ of commodities is higher than the equilibrium price $\tilde{p}_{\alpha+}$ of means of subsistence commodities in the form of labor value, that is $\tilde{p}_{\alpha+}>p_{\alpha+}^{*}$; when $b_{\alpha}$ exceeds the equilibrium point $b_{\alpha}^{*}$ of supply and demand, the supply of means of subsistence commodities exceeds the demand, and the market price $\tilde{p}_{\alpha}$ of commodities is lower than the equilibrium price $\tilde{p}_{\alpha+}$ of means of subsistence commodities in the form of labor value, that is $\tilde{p}_{\alpha+}<p_{\alpha+}^{*}$.

(2) Given that $f_{\tilde{p}}^{(\alpha)}: U_{+} \rightarrow \tilde{p}_{\alpha}$ is a real valued function on $U_{+} \subset B^{n-1}$, then the subset of the Cartesian product $B_{+}^{n-1} \times H_{+}=B_{+}^{n}$ of the quantity space and monetary space of means of subsistence commodities in the form of labor value is

$$
\operatorname{epi} f_{\widetilde{p}}^{(\alpha)}=\left\{(x, h) \in B^{n-1} \times H=B^{n} \mid x \in B^{n-1}, f_{\widetilde{p}}^{\alpha}(x) \leq h\right\}
$$

called the subset of the upper graph of $f_{\tilde{p}}^{(\alpha)}(x)$, which represents the set composed of the market price of means of subsistence commodities in the form of labor value corresponding to the labor value in the production field and the corresponding commodity quantity vectors. According to Theorem 3.1, $D \subset B^{n}$ is a closed convex set of Euclidean metric space. Thus, epi $f_{\widetilde{p}}^{(\alpha)}$ is also a closed convex set.

(3) Given that $f_{\tilde{p}}^{(\alpha)}$ is an inverse proportional function, and the aggregate amount of market price function of any commodity is $b_{\alpha} \tilde{p}_{\alpha}=\varpi_{\alpha}$. According to the property of inverse proportional function, the market price function of any commodity $G(\tilde{p}, b)=f_{\tilde{p}}(b) \times b$ is a constant function, it has

$$
G\left(\tilde{p}_{\alpha}, b_{\alpha}\right)=b_{\alpha} \tilde{p}_{\alpha}=b_{\alpha} \frac{b_{\alpha}^{*}}{b_{\alpha}} \frac{\bar{w}_{\alpha}}{\sum_{\alpha=1}^{n-1} \bar{w}_{\alpha} b_{\alpha}^{*}} M=b_{\alpha}^{*} p_{\alpha}=\varpi_{\alpha}
$$

Thus, the aggregate amount of market price of any $\alpha$ th commodity is equal to the aggregate 
amount of equilibrium price. ${ }^{[10]}$ Q.E.D.

Economic meaning: This theorem (1) and (2) taking the means of subsistence commodities in the form of labor value as example, show that the reason why the market price of commodities is a convex function is that there is unbalanced relationship between supply and demand. At the same time, the upper graph of this commodity market price function is a convex set, because exchange value and equilibrium price are the basis for the formation of commodity market price. Because of this, conclusion (3) shows a complex form that, on the one hand, the actual supply quantity of commodities multiplied by the market price is equal to the equilibrium price; On the other hand, if the market price of any commodity is equal to the equilibrium price, the market supply and demand of each commodity must be in balance. However, in this way, the price of commodities has a duality: first, the market price of commodities is equal to the equilibrium price only in the balanced state of supply and demand; second, in the state of unbalanced supply and demand, although the market price of commodities deviates from the value, that is, is not equal to the equilibrium price, the market price of commodities still has the same total price, that is, the balance sheet can still be maintained. It can be seen that the market price of commodities not only fluctuates up and down around the equilibrium price of commodities, but also shows that under the condition of unbalanced supply and demand of commodities, the conservation theorem of total market price of commodities is still the basis for the market to maintain the state of equivalent exchange. Understanding the duality of commodity market price is the key to understand the market equilibrium theory of new theory of value. The fundamental difference between neoclassical economics and new theory of value is reflected here. In the view of neoclassical economics, the equilibrium price of any commodity decreases strictly monotonically with the increase of quantity. However, in our opinion, the phenomenon of diminishing marginal utility happens to be that the market price of commodities deviates from the equilibrium price. At the same time, the conservation theorem of total market price will not change because the market price deviates from the equilibrium price.

\subsection{Classification and Nonlinear Programming of Commodity Market Price}

The market price of commodities is always expressed through the relationship between supply and demand. The market price of any commodity can be expressed as a pair of concave convex functions, including the concave-convex function in the form of labor value and use

[10] Interestingly, Walras asserts that conclusion (3) of this theorem would be "an egregious error" (1874, p.90). 
value corresponding to the market price of the means of subsistence commodities and the concave-convex function in the form of labor value and use value corresponding to the market price of the means of production products. Therefore, to study the relationship between market supply and demand of commodities under the disequilibrium state is actually to classify the market price, and solve the extremum solutions of the corresponding concave convex function of market price.

Definition 5.4 Let $V$ and $D$ be subsets of the market price space $\tilde{P}^{n-1}$ and the commodity value space $B^{n}$, respectively, and $K$ be the function mapping $V \times D$ to $[-\infty,+\infty]$. We call $K$ as the concave-convex function corresponding to the means of subsistence products in the form of labor value and use value. If for $K(\tilde{p}, b)$, it is a concave function of the market price of $\tilde{p} \in V$, and for each $\tilde{p} \in V$, it is a convex function of the equilibrium price of $b \in D$. The definition of the convex-concave function corresponding to means of production products in the form of labor value and use value is similar. These two kinds of functions are collectively referred to as commodity market price saddle function (the definition of saddle function refers to Rockefeller, 1970, p.346). Therefore, based on the above definition, according to the nature that the labor value belongs to the positive range and the use value belongs to the negative range, we can give the concave-convex function sets $K_{1}$ and $K_{2}$ of the market price of means of subsistence commodities corresponding to the labor value and use value as follows:

$$
\begin{gathered}
K_{1}(\tilde{p}, b)=\left\{\begin{array}{l}
K(\tilde{p}, b), \tilde{p} \in V, b \in D^{-\infty} \\
{[0,+\infty], \tilde{p} \in V_{+}, b \in D_{+}} \\
{[0,-\infty], \tilde{p} \in V_{-}, b \in D_{+}}
\end{array}\right. \\
K_{2}(\tilde{p}, b)=\left\{\begin{array}{l}
K(\tilde{p}, b), \tilde{p} \in V, b \in D \\
{[0,+\infty], \tilde{p} \in V_{+}, b \in D_{-}} \\
{[0,-\infty], \tilde{p} \in V_{-}, b \in D_{-}}
\end{array}\right.
\end{gathered}
$$

$K_{1}$ and $K_{2}$ have been shown in the diagram of commodity market value above.

Definition 5.2 In the case that quality is constant, output and price form a planar cartesian coordinate system:

(1) $b_{\alpha}^{*} \neq 0$, when $b_{\alpha}^{*}>0$, the market price function of commodities is $K_{1}$, that is the market price corresponding to the means of subsistence (a concave-convex function in quadrants I 
and IV in a coordinate system with $b_{\alpha}$ as the horizontal axis and $\tilde{p}_{\alpha}$ as the vertical axis), where when $b_{\alpha}>0$ and $\tilde{p}_{\alpha}>0$, it represents the supplier price corresponding to the labor value (in quadrant I, i.e. the credit in the balance sheet); when $b_{\alpha}>0$ and $\tilde{p}_{\alpha}<0$, it represents the buyer price corresponding to the use value (in quadrant IV, i.e. the debit in the balance sheet).

(2) $b_{\alpha}^{*} \neq 0$, when $b_{\alpha}^{*}<0$, the price of a commodity is the market price corresponding to the means of production, where when $b_{\alpha}<0$ and $\tilde{p}_{\alpha}>0$, it represents the form of use value of the means of production, i.e. the market price of the means of production consumed in the production process (in quadrant II, concave function, i.e. the debit in the balance sheet); when $b_{\alpha}<0$ and $\tilde{p}_{\alpha}<0$, it represents the form of labor value corresponding to the means of production, i.e. the market price converted into production cost in the production process (in quadrant III, convex function, i.e. the credit in the balance sheet).

Proof: According to Definitions 5.1 and 5.2, the market price is an inverse proportional function symmetric on the horizontal axis. It is easy to know that in the inverse proportional function, $b_{\alpha}^{*} \neq 0$, it also has the property of monotonicity. When $b_{\alpha}>0$ and $\tilde{p}_{\alpha}>0$, the image is located in quadrants I, it is a decreasing function; When $b_{\alpha}>0$ and $\tilde{p}_{\alpha}<0$, the image is located in quadrants IV, it is an increasing function; When $b_{\alpha}<0$ and $\tilde{p}_{\alpha}>0$, the image is located in quadrants II, it is an increasing function; When $b_{\alpha}<0$ and $\tilde{p}_{\alpha}<0$, the image is located in quadrants III, it is a decreasing function.

According to the definition of value dimension in the new theory of value, the value of commodities consists of labor value and use value, in which labor value belongs to positive field and use value belongs to negative field. At the same time, labor value is related to the production process, and use value is related to the consumption process (Wu et al., 2020). In addition, according to Axiom 3 in the new theory of value, the value force is equal to the value reaction force, and assets = liabilities ( $\mathrm{Wu}$ et al., 2020) Therefore, for the means of subsistence products, the supplier price belongs to the positive field, and the buyer price belongs to the negative field. For the product of means of production, the product cost in the production process belongs to the positive number field, and the aggregate amount of prices of the consumed means of production belongs to the negative field. Thus, according to the monotonicity of the inverse proportional function, the concave-convex function of market 
price is classified from the above conclusion, and the above conclusion can be attained obviously. Q.E.D.

Economic meaning: In the case that quality is constant and output and price constitute a plane Cartesian coordinate system, the market price of commodities has two pairs and four forms of expression. Understanding this economic relationship will help us to distinguish the market price of commodities in the field of production and consumption, as well as the corresponding labor value and use value in different states of supply and demand under the special economic laws. In the case that quality is not constant, and there are business strategies to improve quality and reduce quality, the market price of commodities will have four pairs, eight forms of expression. Interested readers can prove themselves.

Definition 5.5 Let $B^{n}=B^{n-1} \times H$ denote that $B^{n}$ is the Cartesian product of commodity space $B^{n-1}$ as the carrier of use value and commodity $H \cong R$ as money. Let $x=\left(b_{1}, b_{2}, \cdots, b_{n}\right) \in B^{n-1}$, then the differential price function of a commodity is the absolute value of the difference between the equilibrium price and the market price, i.e

$$
\left.F(b, x)=F\left(f_{p}(b), f_{\tilde{p}}(x)\right)=\mid f_{p}(b)-f_{\tilde{p}}(x)\right)|=| \tilde{p}_{\alpha}-p_{\alpha}|=| \hat{p}_{\alpha} \mid
$$

which indicates the deviation degree between the equilibrium price and the market price of commodities, as well as the non-equilibrium degree of the relationship between supply and demand of commodities. Obviously, $F$ is a generalized function or multivalued function from $P^{n-1}$ to $B^{n}$, which satisfies that for each $b \in B^{n}, f_{p}(b)=p \in P^{n-1}$ has a corresponding market price function $f_{\tilde{p}}(x)$ defined on $B^{n-1}$ and valued in $[0,+\infty]$. The value of $f_{\tilde{p}} \quad$ at point $b \in B^{n} \quad$ is recorded as a composite function $f_{\tilde{p}} \circ f_{p}: B^{n} \rightarrow P^{n-1} \rightarrow \tilde{P}^{n-1}$ to form a new corresponding function

$$
(b, x) \rightarrow\left(F_{p}=f_{\tilde{p}} \circ f_{p}\right)(b), \quad(b, x) \in P^{n-1} \times B^{n}=R^{2 n-1}
$$

called the graph function of $F$. Therefore, the convex programming $(P)$ related to $F$ is a "minimization problem with perturbation", in which the function $F 0$ is the minimum element on $B^{n}$, that is, $f_{p}: B^{n} \rightarrow P^{n-1}$ is differentiable at the rational demand vector $b^{*} \in B^{n}$ then $f_{\tilde{p}} \circ f_{p}: B^{n} \rightarrow P^{n-1} \rightarrow \tilde{P}^{n-1}$ is differentiable at $b^{*}$, so the given perturbation is 
attained by replacing $F 0$ with $f_{\tilde{p}}$ for different $p \in P^{n-1}$. For the specific description of this double correspondence, see the discussion of Theorem 5.5 below.

Definition 5.6 Let the total differential price function of $n-1$ commodities be the absolute value of the sum of the difference of $n-1$ equilibrium prices minus n-1 market prices, i.e

$\left.\left.G\left(f_{p}(b), f_{\tilde{p}}(x), x\right)=\mid \sum_{\alpha=1}^{n-1} f_{p}(b) x-\sum_{\alpha=1}^{n-1} f_{\tilde{p}}(x) x\right)|=| \sum_{\alpha=1}^{n-1} \tilde{p}_{\alpha} b_{\alpha}-\sum_{\alpha=1}^{n-1} p_{\alpha} b_{\alpha}\right)|=| \varpi \mid(5-2-4)$

If set the money supply as $h=\sum_{\alpha=1}^{n-1} \tilde{p}_{\alpha}=\sum_{\alpha=1}^{n-1} \frac{\bar{w}_{\alpha} b_{\alpha}^{*}}{\sum_{\alpha=1}^{n-1} \bar{w}_{\alpha} b_{\alpha}^{*}} h=\sum_{\alpha=1}^{n-1} \lambda_{\alpha}=1$, and $h=\sum_{\alpha=1}^{n-1} p_{\alpha}=\sum_{\alpha=1}^{n-1} \lambda_{\alpha}=1$, then $G$ is a convex function of the aggregate amount of prices defined on the convex hull $S$ if the following conditions are satisfied

$$
S:=\bigcap_{\substack{X \subseteq D \subseteq B^{n} \\ E \text { is the convex hull of equilibrium price, see (42-1) }}} E
$$

where $X$ is the true subset of market price space $\tilde{P}^{n-1}$, and the convex hull of $X$ is constructed by the linear combination of all market price combination points $\left(\tilde{p}_{1}, \tilde{p}_{2}, \cdots, \tilde{p}_{n}\right)$ in $X$, that is

$$
S:=\left\{\sum_{i=1}^{l} \tilde{p}_{i} b_{i} \mid b_{i} \in X, \sum_{\alpha=1}^{n-1} \tilde{p}_{\alpha}=\sum_{\alpha=1}^{n-1} \frac{b_{\alpha}^{*}}{b_{\alpha}} \lambda_{\alpha}=\frac{b_{\alpha}^{*}}{b_{\alpha}} \frac{\bar{w}_{\alpha} b_{\alpha}^{*}}{\sum_{\alpha=1}^{n-1} \bar{w}_{\alpha} b_{\alpha}^{*}} h=1\right\}
$$

Obviously, $S$ is the intersection of equilibrium price convex hull $E$ and market price convex set $X$. Therefore, convex hull $S$ is the problem of minimizing $f_{p}(b)-f_{\tilde{p}}(b)$. Specifically, $f_{p}$ is a normal convex function defined on $D \subset B^{n} ; f_{\tilde{p}}$ has different concave-convex properties in different states where constant $b^{*}$ and independent variable $b$ are positive and negative (see Theorem 5.2 for details). Therefore, this is a general minimization problem on convex hull $S$ including various special cases, that is, under the conditions of (5-2-3), (5-2-4), (5-2-5) and (5-2-6), $f_{p}(b)-f_{\tilde{p}}(b)$ is a convex function defined on $V \times D \subset P^{n-1} \times B^{n}$. The duality of this convex function comes down to the relationship between minimizing the difference function $f_{p}(b)-f_{\tilde{p}}(b)$ and maximizing the 
difference function $f_{p}^{*}(b)-f_{\tilde{p}}^{*}(b)$, where $f_{p}^{*}(b)$ is the (convex) conjugate of $f_{p}$ and $f_{\tilde{p}}^{*}(b)$ is the (concave) conjugate of $f_{\tilde{p}}$. Note that minimizing $f_{p}-f_{\tilde{p}}$ effectively occurs on convex set $\operatorname{dom}\left(f_{p}-f_{\widetilde{p}}\right)=\operatorname{dom} f_{p} \cap \operatorname{dom} f_{\widetilde{p}}$ and maximizing $f_{p}^{*}-f_{\tilde{p}}^{*}$ effectively occurs on convex set $\operatorname{dom}\left(f_{p}^{*}-f_{\widetilde{p}}^{*}\right)=\operatorname{dom} f_{p}^{*} \cap \operatorname{dom} f_{\widetilde{p}}^{*}$.

Lemma 5.2 (Fenchel duality theorem) Let $f_{p}$ be a normal convex function defined on $B^{n}$, and $f_{\tilde{p}}$ be a normal concave function defined on $B^{n-1}$. As long as one of the conditions

(a) $\operatorname{ri}\left(\operatorname{dom} f_{p}\right) \cap \operatorname{ri}\left(\operatorname{dom} f_{\widetilde{p}}\right) \neq \varnothing$;

(b) $f_{p}$ and $f_{\tilde{p}}$ are closed, $\operatorname{ri}\left(\operatorname{dom} f_{p}\right) \bigcap \operatorname{ri}\left(\operatorname{dom} f_{\widetilde{p}}\right) \neq \varnothing$

is valid, then

$$
\inf _{x}\left(f_{p}(b)-f_{\tilde{p}}(x)\right)=\sup _{x^{*}}\left(f_{p}^{*}\left(b^{*}\right)-f_{\tilde{p}}^{*}\left(x^{*}\right)\right)
$$

When condition (a) holds, the supremum inf is attained at some points $x^{*}$, and when condition (b) holds, the infimum sup is attained at some points $x$; If (a) and (b) both hold, the supremum and infimum are necessarily finite, that is, be bounded. If $f_{\tilde{p}}$ is a polyhedron, $\operatorname{ri}\left(\operatorname{dom} f_{\widetilde{p}}\right)$ and $\operatorname{ri}\left(\operatorname{dom} f_{\widetilde{p}}^{*}\right)$ in (a) and (b) can be replaced by $\left(\operatorname{dom} f_{\widetilde{p}}\right)$ and $\left(\operatorname{dom} f_{\widetilde{p}}^{*}\right)$ respectively (because $f_{p}$ and $f_{\tilde{p}}$ are both polyhedral, ri can be omitted) (Rockefeller, 1970, p.327)

Theorem 5.3 (1) If the market price difference function of commodities has the form of (5-2-3) or (5-2-4), the feasible domain of $f_{p}$ and $f_{\tilde{p}}$ is a closed set and differentiable at $\left(x=x^{*}\right)$, that is, when the left derivative and right derivative exist at the same time, there must be

$$
\inf _{x}\left(f_{p}(b)-f_{\tilde{p}}(x)\right)=\sup _{x^{*}}\left(f_{p}^{*}\left(b^{*}\right)-f_{\tilde{p}}^{*}\left(x^{*}\right)\right)
$$

Where when condition (a) of Lemma 5.2 holds, the supremum is attained at the rational demand quantity $b^{*}$, and when condition (b) of Lemma 5.2 holds, the supremum is attained at the rational demand $x^{*}$; If (a) and (b) are true at the same time, the supremum and infimum must be bounded. 
(2) If and only if $F(b)=0$, or $G(b)=0$, the market price of any a commodity is equal to the equilibrium price, i.e. $\tilde{p}_{\alpha}=p_{\alpha}$. At the same time, the supply and demand of commodities in the market is balanced, that is $b_{\alpha}=b_{\alpha}^{*}, \alpha=1,2, \cdots, n-1$.

Proof: (1) First, according to Theorem 4.1, any $\alpha$ th equilibrium price $p_{\alpha}$ is a convex function; according to Theorem 5.1, any $\alpha$ th market price function $\tilde{p}_{\alpha}$ is a normal concave function in the case of $b_{\alpha}^{*}>0$ and $b_{\alpha}>0$. According to Definition (5-1-3), $\frac{b_{\alpha}^{*}}{b_{\alpha}} p_{\alpha}=\tilde{p}_{\alpha}$. Therefore, $\operatorname{ri}\left(\operatorname{dom} f_{p}\right) \cap \operatorname{ri}\left(\operatorname{dom} f_{\widetilde{p}}\right) \neq \varnothing$, that is, when the feasible domain of $p_{\alpha}$ and $\tilde{p}_{\alpha}$ is a closed set and differentiable at $\left(b^{*}, x^{*}\right)$, the intersection of the relative interior of $p_{\alpha}$ and $\tilde{p}_{\alpha}$ is not empty. Obviously, both $F(b, x)$ and $G(b, x)$ satisfy the conditions (a) and (b) of Lemma 5.2. In addition, according to the conclusion that the intersection of convex sets is still a convex set, it is easy to prove that if (5-2-4) is a function of aggregate amount of difference prices defined on convex hull $S$, then (5-2-4) is also a convex function. At the same time, the intersection of the relative interior of $p_{i}$ and $\tilde{p}_{i}$, that is, the convex hull $S$ is not empty.

Second, it is known that any $\alpha$ th market price function $\tilde{p}_{\alpha}$ have different concave-convex properties in the case of $b_{\alpha}^{*}>0$, and $b_{\alpha}>0$, or and $b_{\alpha}<0$; OR $b_{\alpha}^{*}<0$, and $b_{\alpha}>0$, or and $b_{\alpha}<0$. However, any concave convex function is generated under the constraint of inverse proportional function $\frac{b_{\alpha}^{*}}{b_{\alpha}} p_{\alpha}=\tilde{p}_{\alpha}$. Therefore, the conclusion of this theorem (1) is also applicable to the market price $\tilde{p}_{\alpha}$ with the form of (5-2-3) or (5-2-4) of the market price difference function of commodities in any cases.

(2) It is known that the market price of any $\alpha$ th commodity is $\frac{b_{\alpha}^{*}}{b_{\alpha}} p_{\alpha}=\tilde{p}_{\alpha}, b_{\alpha}^{*} \neq 0$, $\alpha=1,2, \cdots, n-1$. Therefore, we know that the market price $\tilde{p}_{\alpha}$ of any commodity is generated by the supply-demand coefficient times the equilibrium price. At the same time, the equilibrium price vector $p \in P^{n-1}$ is known, in which the equilibrium price of any $\alpha$ th commodity is $p_{\alpha}, \alpha=1,2, \cdots, n-1$. Therefore, we can define the fixed point of the commodity market price function $T\left(p_{\alpha}\right)$ of any $\alpha$ th commodity under unbalanced conditions as 


$$
T\left(p_{\alpha}\right)=T\left(f_{\tilde{p}}\left(b_{\alpha}, \lambda_{\alpha}\right)\right)=\frac{b_{\alpha}^{*}}{b_{\alpha}} \frac{\bar{w}_{\alpha}}{\sum_{\alpha=1}^{n-1} \bar{w}_{\alpha} b_{\alpha}^{*}} M=p_{\alpha}
$$

Then, if (5-2-7) is to be established, $b_{\alpha}^{*}-b_{\alpha}=0$ must be made, that is

$$
T\left(p_{\alpha}\right)=\frac{b_{\alpha}^{*}}{b_{\alpha}^{*}} \frac{\bar{w}_{\alpha}}{\sum_{\alpha=1}^{n-1} \bar{w}_{\alpha} b_{\alpha}^{*}} H=p_{\alpha} \Rightarrow b_{\alpha} \tilde{p}_{\alpha}=b_{\alpha}^{*} \bar{w}_{\alpha}=p_{\alpha}
$$

So, the necessity exists. Further, let $b_{\alpha} \rightarrow b_{\alpha}^{*}$ and $-b_{\alpha} \rightarrow b_{\alpha}^{*}$ and substitute (5-2-8), then there is

$$
\lim _{b_{\alpha} \rightarrow b_{\alpha}^{*},-b_{\alpha} \rightarrow b_{\alpha}^{*}} T\left(p_{\alpha}\right)=\tilde{p}_{\alpha}=\frac{b_{\alpha}^{*}}{b_{\alpha}} p_{\alpha}=p_{\alpha} \Rightarrow \tilde{p}_{\alpha} b_{\alpha}=p_{\alpha}
$$

Therefore, when the supply and demand of commodities are balanced, the market price of commodities is equal to the equilibrium price, which has sufficiency. In particular, when $\tilde{p}_{\alpha}=p_{\alpha}$, it is obviously impossible to make $\left(b_{\alpha} \neq b_{\alpha}^{*}\right)$ under the constraints of $f_{p}$ and $f_{\tilde{p}}$.

In addition, in the case of $G(b, x)$, it can be seen from definition 5.6 that $G$ is a convex function of the aggregate amount of prices defined on the convex hull of the equilibrium price. Therefore, from the properties that the intersection of convex sets is still convex sets, and the intersection of convex functions defined on convex sets is still convex functions on convex hulls, it is obvious that the conclusion of this theorem (2) is also valid. Q.E.D.

Economic meaning: This theorem analyzes the commodity price corresponding to labor value, that is, the market equilibrium of the buyer's price and the seller's price corresponding to the equilibrium price and the market price. This problem is the core issue concerned by neoclassical economics since the marginal revolution. Here, we give different theoretical conclusions from the mainstream general equilibrium theory: (1) the equilibrium price of commodities is determined by the maximum value of the commodity value function. Among the complex variable functions of value, the complex variable function of value satisfying the harmonic condition determines the maximum of the value of each commodity. (See Theorem 2.2) When the quality is constant, it is the equilibrium price determined by the affine mapping of the exchange value of N-1 commodities in the balanced state of supply and demand. (See Theorem 3.2) So the equilibrium price is uniquely determined. (2) The market price of 
commodities is a convex function and has the property of diminishing marginal value. It is only a special manifestation that the market price of commodities deviates from the equilibrium price under the condition of unbalanced supply and demand, not the general economic law. (3) The market price of commodities has an equilibrium price fixed point, that is, there is an extremum solution under "bounded and continuous" conditions. Therefore, the equilibrium price exists only under special conditions, while in general, the price of commodities is disequilibrium.

\subsection{Stability Analysis of n-1 Ordinary Differential Equations of Commodity Market Value}

Above this section, the economic problems of commodity value and price in the equilibrium and nonequilibrium relationship between supply and demand in static and stable economic systems are discussed respectively. Now we further investigate the problem of ordinary differential equations of commodity value in the critical state of dynamic and unstable systems. In this case, the stability theory of Lyapunov and Poincare in theoretical mechanics can become the theoretical paradigm of our research problems.

\section{(1) First-order Ordinary Differential Equation of Commodity Market Value}

Assuming that the ordinary differential equation of commodity market value is a system of ordinary differential equations with time as the parameter and independent variable, the space composed of the unknown function of the commodity price difference function can be called the commodity phase space, the trajectory of the solution of the value differential equation in the commodity phase space is called the trajectory line, and the trajectory line can also be defined as the projection of the integral curve in the phase space. Making time single parameter curve family

$$
V(x(t))=c \quad(c>0)
$$

where $x \in B^{n}$, the norm of $x$ is $\|x\|=\sqrt{\sum_{\alpha=1}^{n} x^{2}}$.

Let $V(x(t))$ be a positive definite function, i.e. $V(0)=0$, and when $x \neq 0, V(x(t))>0$ and $V(x(t))$ is continuous; when $c$ is small enough, the curve family (5-3-1) is closed, and when $c_{1}<c_{2}$, the closed curve $V=c_{1}$ is wholly included in the closed curve $V=c_{2}$.

If the ordinary differential equation of commodity market value is a positive definite function, it means that the initial state of the problem we study is that the differential price function of 
commodity is a number greater than zero, that is, when $x \neq 0, V(x(t))>0$.

So, as time goes on, if there is $\frac{\mathrm{d} V}{\mathrm{~d} t} \leq 0$ along the trajectory line $x(t)$, it means that the function $V(x(t))$ is a non increasing function of $t$ for all $t \geq t_{0}$. Therefore, as $t \geq t_{0}$ increases, any trajectory line $x(t)$ will enter the closed curve family (5-3-1) layer by layer or move along these curves (the whole curve or a section). It will never go from the inside of any closed curve of (5-3-1) to the outside.

In this way, for any given positive number $\varepsilon<H$, make the maximum closed curve $V(x(t))=c$ in domain $\|x\| \leq \varepsilon$, and take the maximum inscribed circle centered on the origin in this closed curve, and its radius is recorded as $\delta$. Then the trajectory line starting from any point $x_{\delta}$ in domain $\|x\| \leq \delta$ always stays within $V=c$, and naturally stays within domain $\|x\| \leq \varepsilon$, that is, $\|x(t)\|<\varepsilon$, so the zero solution is stable.

On the contrary, when $\frac{\mathrm{d} V}{\mathrm{~d} t}$ is definite negative, only the trajectory line $x(t)$ is allowed to enter the curve family (5-3-1) from the outside to the inside and asymptotically approach the origin $x=0$, and the trajectory line is not allowed to hover on any curve in (5-3-1). At this time, the zero solution is asymptotically stable.

The unstable situation can be discussed similarly. At this time, the trajectory will leave the curve family (5-3-1) from the inside out and go out of the total commodity value domain $\|x\| \leq W$.

\section{(2) Quadratic Ordinary Differential Equation of Commodity Market Value}

Next, for the above (5-3-1) problem, we further discuss the construction of quadratic V-function for linear differential equations with constant coefficients, and investigate how to apply $\mathrm{v}$ function to determine the stable state of the solution of nonlinear differential equations. For simplicity, we consider only nonlinear stationary differential equations

$$
\frac{\mathrm{d} x}{\mathrm{~d} t}=f(x) 。
$$

Suppose $f(0)=0$, and $f(x)$ has a continuous partial derivative in a domain $G:\|x\| \leq A(A$ is a positive constant), so the solution determined by the initial value condition $x\left(t_{0}\right)=x_{0}$ of the system of equations (6.20) exists and is unique in domain $G$. Obviously, $x=0$ is its 
particular solution.

Definition 5.7 Assume that the ordinary differential equation $V(x(t))$ of commodity market value is a real continuous function defined in domain $\|x\| \leq W, V(0)=0$. If there is always $V(x(t)) \geq 0$ in this domain, the function $V$ is called constant positive; If there is $V(x(t))>0$ for all $x \neq 0$, the function $V$ is called definite positive; If the function $-V$ is definite positive (or constant positive), it is called $V$ definite negative (or constant negative). Further, assuming that the partial derivatives of the function $V(x(t))$ with respect to all variables exist and are continuous, substitute the solution of equation (6-1), and then find the derivative for $t$

$$
\frac{\mathrm{d} V}{\mathrm{~d} t}=\sum_{i=1}^{n} \frac{\partial V}{\partial x_{i}} \frac{\mathrm{d} x_{i}}{\mathrm{~d} t}=\sum_{i=1}^{n} \frac{\partial V}{\partial x_{i}} f_{i}
$$

The derivative $\frac{\mathrm{d} V}{\mathrm{~d} t}$ attained is called the total derivative of function $V$ through equation $(5-3-2)$

Lemma 5.3 If the first order linear differential equations

$$
\frac{\mathrm{d} x}{\mathrm{~d} t}=A x
$$

whose eigenvalues $\lambda_{i}$ do not satisfy the relation $\lambda_{i}+\lambda_{j}=0(i, j=1,2, \cdots, n)$, then there is a unique quadratic form for any negative definite (or positive definite) symmetric matrix $C$

$$
V(x)=x^{T} B x \quad\left(B^{T}=B\right)
$$

Let it pass through the total derivative of the system of equations (5-3-2)

$$
\frac{\mathrm{d} V}{\mathrm{~d} t}=x^{T} C x \quad\left(C^{T}=C\right)
$$

and the symmetric matrix $B$ satisfies the relation

$$
A^{T} B+B A=C
$$

Here $A^{T}, B^{T}, C^{T}, x^{T}$ denotes the transpose of $A, B, C, x$, respectively. (Wang, et al., 2006, p.271-272)

Lemma 5.4 If a definite positive function $V(x(t))$ can be found for the system of differential 
equations (5-3-2), and its total derivative $\frac{\mathrm{d} V}{\mathrm{~d} t}$ through (5-3-3) is a constant negative function or equal to zero, the zero solution of the system of equations (5-3-3) is stable.

If there is a definite positive function $V(x(t))$ whose total derivative $\frac{\mathrm{d} V}{\mathrm{~d} t}$ through (5-3-2) is definite negative, the zero solution of the system of equations (5-3-2) is asymptotically stable.

If there is a function $V(x(t))$ and a non-negative constant $\mu$, the total derivative $\frac{\mathrm{d} V}{\mathrm{~d} t}$ through (5-3-2) can be expressed as

$$
\frac{\mathrm{d} V}{\mathrm{~d} t}=\mu V+W(x)
$$

And when $\mu=0, W$ is a definite positive function, while when $\mu \neq 0, \mathrm{~W}$ is a constant positive function or equal to zero; If there is at least some $\bar{x}$ in any small neighborhood of $x=0$, so that $V(\bar{x}(t))>0$, then the zero solution of the system of equations (5-3-2) is unstable. (Wang, et al., 2006, p.246-247)

Theorem 5.4 (1) If the total derivative $\frac{\mathrm{d} V}{\mathrm{~d} t}$ of the ordinary differential equation of commodity market value through (5-3-2) is a constant negative function or equal to zero, the zero solution of the equation system (5-3-2) is stable;

(2) If $V(x(t))$ is a definite positive function and its total derivative $\frac{\mathrm{d} V}{\mathrm{~d} t}$ through (5-3-2) is definite negative, the zero solution of the system of equations (5-3-2) is asymptotically stable; (3) If $V(x)$ is a negative positive function and there is a non negative constant $\mu$, the total derivative $\frac{\mathrm{d} V}{\mathrm{~d} t}$ through (5-3-2) can be expressed as

$$
\frac{\mathrm{d} V}{\mathrm{~d} t}=\mu V+W(x)
$$

and when $\mu=0, W$ is a definite positive function, while when $\mu \neq 0, W$ is a constant positive function or constantly equal to zero;

(4) If there is at least some $\bar{x}$ in any small neighborhood of $x=0$, so that $V(\bar{x}(t))>0$, then the zero solution of the system of equations (5-3-2) is unstable. 
Proof: Directly proved by Lemma 5.3 and 5.4.

Economic meaning: This theorem shows that the stability criterion of the ordinary differential equation of commodity market value is very clear: with the extension of time, if the ordinary differential equation of commodity market value is a decreasing function, then the market price is stable; On the other hand, if the ordinary differential equation of the market value of commodities is an increasing function, then there is no stability of the market price. Obviously, there are two corollaries to this theorem:

(1) The stability judgment is based on the Euclidean metric space of commodity value. In this case, commodity value and price theory have a total order relationship as the basis of theoretical discussion. Therefore, this theorem is incompatible with Arrow's impossibility theorem. That is to say, as long as money does not smell, then, no matter how strong the will of personal preference can not resist that the price of social wealth will return to the value.

(2) Market equilibrium is not absolute, but relative. That is to say, if most people understand the objective economic law and can consciously eliminate the possible economic crisis caused by excess capacity, the stability of market equilibrium will exist. On the contrary, if the majority of people violate the objective economic law, resulting in the economic crisis of excess capacity, the market will not be stable, even though the Pareto optimal allocation of social resources has been achieved at this time.

\section{References}

[1] Aristotle. Politics, translated by Benjamin Jowett. Kichener: Batoche Books, 1999.

[2] Arrow, K. J. Social Choice and Individual Values (1951) 2nd ed. New Heaven, CT: Cowles Foundation, Yale University, 1970

[3] Arrow, K. J., \& Debreu, G. Existence of an Equilibrium for a Competitive Economy, Econometrica, 1954, Vol. 22, No. 3, 265-290

[4] Arrow, K. J., Intriligator M. D. Handbook of Mathematical Economics, Volume 1 (1981). North Holland, 1987)

[5] Banach, S. Sur les opérations dans les ensembles abstraits et leur application aux équations intégrales. Fund. Math. 1922, 3: 133-181.

[6] Debreu, G. Theory of Value: An Axiomatic Study of Economic Equilibrium. New York: 
Wiley, 1959.

[7] Hicks, J. R. \& Allen, R. G. D. A Reconsideration of the Theory of Value. Economica, 1934, 1(1), 196-219.

[8] Jeffery, A. Advanced Engineering Mathematics. Harcourt/Academic Press, 2002.

[9] Jevons, W. S. The Theory of Political Economy (1871), 5th ed. New York, Sentry Press, 1965

[10] Marx, K. 1867. Das Capital, Vol.I, in Marx and Engels: Collected Works, Vol. 35. London, Lawrence \& Wishart (1996)

[11] Nash, J. Non-Cooperative Games. The Annals of Mathematics, 1951, 54: 286-295.

[12] Pareto, V. Manuel d' Economic Politique (Manual of Political Economy) deuxieme edition (1906). Paris, M. Giard, 1927.

[13] Richardo, D. On the Principles of Political Economy and Taxation (1817). Kitchener, Batoche Books, 2001

[14] Rockafellar, R. T. Convex analysis. Princeton: Princeton University Press, 1970.

[15] Smith, A. An Inquiry into the Nature and Causes of the Wealth of Nations (1776). New York, Random House, 1994

[16] Neumann, J. V. , Morgenstern, O. Theory of Games and Economic Behavior. Princeton University Press. 1953

[17] Walras, L. Elements of Pure Economics (1874). Translated by W. Jaffe, London: Allen and Unwin (1954)

[18] Wang J.H. Introduction to Topology, Shanghai Science and Technology Press, 1978

[19] Wang G., Zhou Z., Zhu S., Wang S. et al. Ordinary differential equations (3rd Ed.). Beijing: Higher Education Press, 2006

[20] Wu, J., Makarov, V. L., Bakhtizin A.R., Wu, Z. et al. The New Theory of Value, Economi cs and Mathematical Methods, 2020, 56(4):5-19.

[21] Yang, X. \& Borland, J. A Microeconomic Mechanism for Economic Growth, Journal of Political Economy, 1991, vol. 99, no. 3, 460-482.

[22] Yu, J.R. Complex Variable Function (3rd edition). Beijing: Higher Education Press. 2000 